\title{
RATIONAL SURFACES WITH A LARGE GROUP OF AUTOMORPHISMS
}

\author{
SERGE CANTAT AND IGOR DOLGACHEV
}

\section{Contents}

1. Introduction

2. Halphen surfaces

3. Coble surfaces

4. Gizatullin's Theorem and Cremona special point sets of nine points

5. The general case

6. Cremona special sets of $n \geq 10$ points lying on a cubic curve 893

7. Non-algebraically closed fields and other surfaces 900

Acknowledgement

References

\section{INTRODUCTION}

Let $\mathbb{K}$ be an algebraically closed field, and $X$ be a projective surface defined over $\mathbb{K}$. The group of automorphisms $\operatorname{Aut}(X)$ acts on the Néron-Severi group of $X$. This action preserves the intersection form and the canonical class $K_{X}$, and therefore provides a morphism from $\operatorname{Aut}(X)$ to the group of integral isometries $\mathrm{O}\left(K_{X}^{\perp}\right)$ of the orthogonal complement $K_{X}^{\perp}$. When $X$ is rational, the image satisfies further constraints: It is contained in an explicit Coxeter subgroup $W_{X}$ of $\mathrm{O}\left(K_{X}^{\perp}\right)$, and $W_{X}$ has infinite index in $\mathrm{O}\left(K_{X}^{\perp}\right)$ as soon as the rank $\rho(X)$ of the Néron-Severi group of $X$ exceeds 11.

A natural problem is to describe all projective surfaces $X$ for which $\operatorname{Aut}(X)$ is infinite and its image in this orthogonal or Coxeter group is of finite index. When $\mathbb{K}$ is the field of complex numbers, the problem asks for a classification of complex projective surfaces with maximal possible groups of isotopy classes of holomorphic diffeomorphisms.

We solve this problem when $X$ is a rational surface. It turns out that this is the most interesting and difficult case. In Section 7.2 we briefly discuss other types of surfaces; one can treat them by more or less standard arguments.

Received by the editors July 5, 2011 and, in revised form, December 9, 2011, and January 23, 2012.

2010 Mathematics Subject Classification. Primary 14E07, 14J26, 14J50, 20F55, 32H50. 
1.1. Automorphisms of rational surfaces. Let $X$ be a rational surface defined over $\mathbb{K}$. The Néron-Severi group of $X$ coincides with the Picard group $\operatorname{Pic}(X)$; its rank $\rho(X)$ is the Picard number of $X$. We denote by $\operatorname{Aut}(X)^{*}$ the image of $\operatorname{Aut}(X)$ in the orthogonal group $\mathrm{O}(\operatorname{Pic}(X))$. There are two alternative possibilities for $\operatorname{Aut}(X)$ to be infinite.

The first occurs when the kernel $\operatorname{Aut}(X)^{0}$ of the action of $\operatorname{Aut}(X)$ on $\operatorname{Pic}(X)$ is infinite. In this case, $\operatorname{Aut}(X)^{0}$ is a linear algebraic group of positive dimension and $\operatorname{Aut}(X)^{*}$ is a finite group (see 25]). All such examples are easy to describe because the surface $X$ is obtained from a minimal rational surface by a sequence of Aut $(X)^{0}$-equivariant blowups. Toric surfaces provide examples of this kind.

In the second case, the group $\operatorname{Aut}(X)^{*}$ is infinite, $\operatorname{Aut}(X)^{0}$ is finite, and then $X$ is obtained from the projective plane $\mathbb{P}^{2}$ by blowing up a sequence of points $p_{1}, \ldots, p_{n}$, with $n \geq 9$ (see [35]). The existence of such an infinite group $\operatorname{Aut}(X)^{*}$ imposes drastic constraints on the point set $\mathcal{P}=\left\{p_{1}, \ldots, p_{n}\right\}$ and leads to nice geometric properties of this set. There are classical examples of this kind as well as very recent constructions (see [20, 3, 31, 40]). Our goal is to classify point sets $\mathcal{P}$ for which the group $\operatorname{Aut}(X)^{*}$ is the largest possible, in a sense which we now make more precise.

1.2. The hyperbolic lattice. Let $\mathbb{Z}^{1, n}$ denote the standard odd unimodular lattice of signature $(1, n)$. It is generated by an orthogonal basis $\left(\mathbf{e}_{0}, \mathbf{e}_{1}, \ldots, \mathbf{e}_{n}\right)$ with

$$
\mathbf{e}_{0}^{2}=1, \quad \text { and } \quad \mathbf{e}_{i}^{2}=-1 \quad \text { for } \quad i \geq 1 .
$$

The orthogonal complement of the vector

$$
k_{n}=-3 \mathbf{e}_{0}+\left(\mathbf{e}_{1}+\cdots+\mathbf{e}_{n}\right)
$$

is a sublattice $\mathbb{E}_{n} \subset \mathbb{Z}^{1, n}$. A basis of $\mathbb{E}_{n}$ is formed by the vectors

$$
\boldsymbol{\alpha}_{0}=\mathbf{e}_{0}-\mathbf{e}_{1}-\mathbf{e}_{2}-\mathbf{e}_{3} \text {, and } \boldsymbol{\alpha}_{i}=\mathbf{e}_{i}-\mathbf{e}_{i+1}, i=1, \ldots, n-1 .
$$

The intersection matrix $\left(\boldsymbol{\alpha}_{i} \cdot \boldsymbol{\alpha}_{j}\right)$ is equal to $\Gamma_{n}-2 I_{n}$, where $\Gamma_{n}$ is the incidence matrix of the graph $T_{2,3, n-3}$ from Figure 1. In particular, each class $\boldsymbol{\alpha}_{i}$ has selfintersection -2 and determines an involutive isometry of $\mathbb{Z}^{1, n}$ by

$$
\boldsymbol{s}_{i}: x \mapsto x+\left(x \cdot \boldsymbol{\alpha}_{i}\right) \boldsymbol{\alpha}_{i}
$$

By definition, these involutions generate the Coxeter (or Weyl) group $W_{n}$.

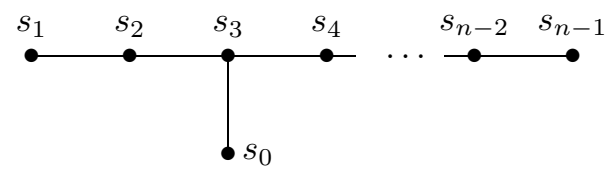

FIGURE 1. Coxeter-Dynkin diagram of type $T_{2,3, n-3}$

1.3. Automorphisms and Coxeter groups. From now on, $X$ is a rational surface for which $\operatorname{Aut}(X)^{*}$ is infinite. We write $X$ as the blowup of $\mathbb{P}^{2}$ at $n$ points $p_{1}$, $\ldots, p_{n}$ with $n \geq 9$; some of them can be infinitely near points and, by convention, $j \geq i$ if $p_{j}$ is infinitely near $p_{i}$. We now describe known constraints on the structure of the group $\operatorname{Aut}(X)^{*}$.

A basis $\left(e_{0}, \ldots, e_{n}\right)$ of $\operatorname{Pic}(X)$ is obtained by taking for $e_{0}$ the class of the total transform of a line in $\mathbb{P}^{2}$, and for $e_{i}, 1 \leq i \leq n$, the class of the total transform of 
the exceptional divisor obtained by blowing up $p_{i}$; in particular, the Picard number $\rho(X)$ is equal to $n+1$. This basis is orthogonal with respect to the intersection form: $e_{0}^{2}=1, e_{i}^{2}=-1$ for $i \geq 1$, and $e_{i} \cdot e_{j}=0$ if $i \neq j$. We call such a basis of $\operatorname{Pic}(X)$ a geometric basis. A geometric basis makes $\operatorname{Pic}(X)$ isometric to the lattice $\mathbb{Z}^{1, n}$, by an isometry which maps $e_{i}$ to $\mathbf{e}_{i}$. Under this isomorphism, the canonical class

$$
K_{X}=-3 e_{0}+e_{1}+\cdots+e_{n}
$$

is mapped to the element $k_{n} \in \mathbb{Z}^{1, n}$. We denote by $\left(\alpha_{i}\right)$ the basis of $K_{X}^{\perp}$ corresponding to $\left(\boldsymbol{\alpha}_{i}\right)$ under this isomorphism, i.e.

$$
\alpha_{0}=e_{0}-e_{1}-e_{2}-e_{3}, \alpha_{1}=e_{1}-e_{2}, \ldots, \alpha_{n-1}=e_{n-1}-e_{n},
$$

and by $s_{i}$ the involutive isometry of $\operatorname{Pic}(X)$ which is conjugate to $s_{i}$. By definition, the group $W_{X}$ is the group of isometries of $\operatorname{Pic}(X)$ generated by these $n$ involutions; thus, $W_{X}$ is isomorphic to the Coxeter group $W_{n}$. It is known that the group $W_{X}$ does not depend on a choice of a geometric basis (see 17, Theorem 5.2, page 27).

The group $\operatorname{Aut}(X)^{*}$ acts by isometries on $\operatorname{Pic}(X)$ and preserves the canonical class. According to Kantor-Nagata's theorem (see [27, Theorem XXXIII, 35], p. 283, or [17, Theorem 5.2), the group $\operatorname{Aut}(X)^{*}$ is contained in $W_{X}$. Thus, we get a series of inclusions

$$
\operatorname{Aut}(X)^{*} \subset W_{X} \subset \mathrm{O}\left(\operatorname{Pic}(X) ; K_{X}\right) \subset \mathrm{O}(\operatorname{Pic}(X)),
$$

where $\mathrm{O}(\operatorname{Pic}(X))$ is the orthogonal group of $\operatorname{Pic}(X)$ with respect to the intersection form, and $\mathrm{O}\left(\mathrm{Pic}(X) ; K_{X}\right)$ is the stabilizer of the canonical class $K_{X}$.

1.4. Cremona special point sets. When $n \leq 8, W_{n}$ is a finite group. We say that the point set $\mathcal{P}:=\left\{p_{1}, \ldots, p_{n}\right\}$ is Cremona special if $n \geq 9$ and the group $\operatorname{Aut}(X)^{*}$ has finite index in $W_{X} 1$ A rational surface obtained by blowing up a Cremona special set will be called Cremona special. In this sense, Cremona special surfaces with fixed Picard number are rational surfaces with largest possible discrete automorphism groups among all rational surfaces with the same rank of the Picard group.

Our goal is to classify Cremona special point sets, a problem that has already been mentioned by Arthur Coble in his book [10, p. 278 (see also 20] and 25]).

Two kinds of such sets have been known since the beginning of the last century. They are general Halphen sets of 9 points and general Coble sets of 10 points (see [10]). Brian Harbourne showed in [24 that, in characteristic $p>0$, and for any integer $n \geq 9$, a general set of $n$ nonsingular points on an irreducible cuspidal cubic curve is Cremona special; for this, he employed the fact that all such points are $p$-torsion points in the group law on the set of nonsingular points on the cubic curve. When $n=9$, Harbourne sets are particular cases of Halphen sets.

We discuss the geometry of Halphen and Coble point sets in Sections 2 and 3. prove that the general ones are indeed Cremona special, and describe precisely what "general" means in this context. By definition, the point set is unnodal if its blowup does not contain smooth rational curves with self-intersection equal to -2 (also called nodal or $(-2)$-curves). This terminology is borrowed from the theory of Enriques surfaces, where it is known that the isomorphism classes of unnodal surfaces form an open subset in the moduli space. A surface obtained by blowing up an unnodal set is called unnodal. We show that unnodal sets form an open

\footnotetext{
${ }^{1}$ Coble called such subsets special, so we somewhat deviate from his terminology.
} 
Zariski subset in the variety of point sets defining Halphen and Coble surfaces, and that Cremona special Halphen and Coble surfaces are exactly the unnodal ones.

Harbourne examples are defined at the beginning of Section 5 and are discussed in Section 6.1

Then, our main result shows that the examples constructed by Halphen, Coble, and Harbourne exhaust all possibilities of Cremona special point sets. As a corollary, if a point set $\mathcal{P}$ is Cremona special, then it is unnodal.

Main Theorem. Let $\mathbb{K}$ be an algebraically closed field. Let $\mathcal{P}$ be a Cremona special point set in $\mathbb{P}_{\mathbb{K}}^{2}$. Then $\mathcal{P}$ is unnodal and one of the following cases occurs:

- $n=9$ and $\mathcal{P}$ is a Halphen set;

- $n=10$ and $\mathcal{P}$ is a Coble set;

- $n \geq 10$, $\operatorname{char}(\mathbb{K})>0$, and $\mathcal{P}$ is a Harbourne set.

Conversely, any such unnodal set is Cremona special.

As a corollary, if a rational surface $X$ is Cremona special, then $-K_{X}$ or $-2 K_{X}$ is effective.

Remark 1.1. a. As explained in Section 7 , there is a stronger version of this theorem which does not assume that $\mathbb{K}$ is algebraically closed, but this requires a careful definition of Cremona special point sets. Nonrational surfaces are dealt with in Section 7.2

1.1. b. When $W_{X}$ is infinite, it is Zariski dense in the real algebraic group $\mathrm{O}\left(K_{X}^{\perp} \otimes \mathbb{R}\right)$. Thus, a natural question is the following. If $X$ is a rational surface and $\operatorname{Aut}(X)^{*}$ is infinite and Zariski dense in $\mathrm{O}\left(K_{X}^{\perp} \otimes \mathbb{R}\right)$, does it follow that $X$ is Cremona special? In other words, is it possible to generalize our Main Theorem under the weaker assumption that $\operatorname{Aut}(X)^{*}$ is infinite and is Zariski dense in $\mathrm{O}\left(K_{X}^{\perp} \otimes \mathbb{R}\right)$ ? Since $W_{9}$ contains a finite index, free abelian group of rank 8 , every Zariski dense subgroup of $W_{9}$ has finite index. Thus, the problem concerns rational surfaces with Picard number at least 10.

1.1. c. There is a notion of Cremona special point sets in projective spaces of higher dimension and in their Cartesian products. Interesting examples of such sets are known (see [10, [20], 19]). Unfortunately, the methods of this paper are specific to dimension 2 and do not extend to the higher-dimensional case.

1.5. An action of $W_{n}$ on point sets and its periodic orbits. Consider the variety $\left(\mathbb{P}^{2}\right)^{n}$ and the diagonal action of $\mathrm{PGL}_{3}$ on it. Consider the GIT-quotient $\mathrm{P}_{n}^{2}$ of the action. It turns out that the group $W_{n}$ acts on $\mathrm{P}_{n}^{2}$ by birational transformations; this Cremona action is described in chapter VI of [20].

Let $\Gamma$ be a subgroup of $W_{n}$. Let $\left(p_{1}, \ldots, p_{n}\right)$ be an ordered stable set of distinct points representing a point $\mathrm{p} \in \mathrm{P}_{\mathrm{n}}^{2}$. Let $X_{\mathrm{p}}$ be the surface obtained by blowing up the projective plane at $p_{1}, \ldots, p_{n}$; its isomorphism class depends only on $\mathrm{p}$. The group $\operatorname{Pic}\left(X_{\mathrm{p}}\right)$ is isomorphic to $\mathbb{Z}^{1, n}$, with an isomorphism depending only on $\mathrm{p}$; we fix such an isomorphism, and the corresponding isomorphism between $W_{X}$ and $W_{n}$.

If $\mathrm{p}$ is contained in the domain of definition of $\gamma$ and $\gamma(\mathrm{p})=\mathrm{p}$ for all $\gamma$ in $\Gamma$, then there exists a subgroup $\Gamma^{\prime} \subset \operatorname{Aut}\left(X_{\mathrm{p}}\right)$ such that the action of $\Gamma^{\prime}$ on $\operatorname{Pic}\left(X_{\mathrm{p}}\right)$ and the identification $W_{n} \sim W_{X_{\mathrm{p}}}$ provide an isomorphism $\Gamma^{\prime} \rightarrow \Gamma$. In other words, points $\mathrm{p} \in \mathrm{P}_{n}^{2}$ which are fixed by the group $\Gamma$ correspond to rational surfaces on which $\Gamma$ is represented by a subgroup of $\operatorname{Aut}\left(X_{\mathrm{p}}\right)$. 
Thus, our Main Theorem classifies periodic orbits of the group $W_{n}$, for $n \geq 9$, i.e. for infinite Coxeter groups $W_{n}$. This provides a dynamical interpretation of the Main Theorem in terms of birational actions of Coxeter groups.

Remark 1.2. There are other nice examples of algebraic dynamical systems for which periodic orbits are related to the construction of interesting geometric objects.

One of them is given by Thurston's pull-back map. If $F: S^{2} \rightarrow S^{2}$ is a (topological) orientation-preserving branched covering map of the sphere $S^{2}$ with a finite post-critical set2 $\mathcal{P}_{F}$ of cardinality $n$, one can ask whether $F$ is equivalent to a holomorphic endomorphism $f$ of the Riemann sphere $\mathbb{P}^{1}(\mathbb{C})$, in the sense that

$$
F=\phi \circ f \circ \psi,
$$

where $\phi$ and $\psi$ are homeomorphisms which are isotopic relative to $\mathcal{P}_{F}$. The map $\sigma_{F}$ defined by Thurston acts on the Teichmüller space of $S^{2}$ with $n$ marked points; fixed points of $\sigma_{F}$ correspond to holomorphic structures on the sphere for which $F$ is realized by an endomorphism $f$. This situation is similar to the one studied here, with $\sigma_{F}$ in place of $W_{n}$ and the Teichmüller space replacing $\mathrm{P}_{n}^{2}$. We refer to 22$]$ for a precise description of Thurston's construction.

Another similar situation, with the mapping class group of a surface $\Sigma$ (in place of $W_{n}$ ) acting on the character variety of the fundamental group $\pi_{1}(\Sigma)$ (in place of $\mathrm{P}_{n}^{2}$ ) is related to hyperbolic structures on three-dimensional manifolds, and to algebraic solutions of Painlevé's sixth equation (see [7] and the references therein).

\section{HALPHEN SURFACES}

In this section, we describe Halphen surfaces, Halphen pencils of genus 1 curves, and their associated point sets. We then show that unnodal Halphen point sets are Cremona special. Most results in this section are known to experts, but may be hard to find in the literature, and will be used in the following sections.

We assume some familiarity with the theory of elliptic fibrations over fields of arbitrary characteristic and refer to [12, Chapter V, for this topic.

2.1. Halphen surfaces of index $m$. By definition, a $(-n)$-curve on a smooth projective surface $X$ is a smooth rational curve with self-intersection $-n$. The genus formula shows the following.

Lemma 2.1. Let $X$ be a smooth projective surface. Let $n$ and $l$ be positive integers.

(1) Assume $-K_{X}$ is nef. If $E$ is $a(-n)$-curve, then $n=1$, or $n=2$ and $E \cdot K_{X}=0$.

(2) Assume that the linear system $\left|-l K_{X}\right|$ contains a reduced, irreducible curve $C$ with $C^{2}<0$. If $E$ is $a(-n)$-curve, then $n=1$, or $n=2$ and $E \cdot C=0$, or $E=C$.

A smooth rational projective surface $X$ is a Halphen surface if there exists an integer $m>0$ such that the linear system $\left|-m K_{X}\right|$ is of dimension 1 , has no fixed component, and has no base point. The index of a Halphen surface is the smallest possible value for such a positive integer $m$.

Let $X$ be a Halphen surface of index $m$. Then $K_{X}^{2}=0$ and, by the genus formula, the linear system $\left|-m K_{X}\right|$ defines a genus 1 fibration $f: X \rightarrow \mathbb{P}^{1}$, which is elliptic

\footnotetext{
${ }^{2}$ The post-critical set is the union of the images of the set of critical points of $f$ under positive iterations of $f$.
} 
or quasi-elliptic if $\operatorname{char}(\mathbb{K})=2$ or 3 . This fibration is relatively minimal in the sense that there is no $(-1)$-curve contained in a fiber.

Proposition 2.2. Let $X$ be a smooth projective rational surface. Let $m$ be a positive integer. The following four properties are equivalent:

(i) $X$ is a Halphen surface of index $m$;

(ii) $\left|-K_{X}\right|$ is nef and contains a curve $F_{0}$ such that $\mathcal{O}_{F_{0}}\left(F_{0}\right)$ is of order $m$ in $\operatorname{Pic}\left(F_{0}\right)$;

(iii) there exists a relatively minimal elliptic or quasi-elliptic fibration $f: X \rightarrow$ $\mathbb{P}^{1}$; it has no multiple fibers when $m=1$ and a unique multiple fiber, of multiplicity $m$, when $m>1$;

(iv) there exists an irreducible pencil of curves of degree $3 m$ with 9 base points of multiplicity $m$ in $\mathbb{P}^{2}$, such that $X$ is the blowup of the 9 base points and $\left|-m K_{X}\right|$ is the proper transform of this pencil (the base point set may contain infinitely near points).

In the proof of (iii) $\Rightarrow$ (iv) below, the classification of minimal rational surfaces is used. Recall that a minimal rational surface is isomorphic to $\mathbb{P}^{2}$ or to one of the Segre-Hirzebruch surfaces $\mathbf{F}_{n}=\mathbb{P}(\mathcal{O} \oplus \mathcal{O}(-n))^{3}$ with $n \geq 0$ and $n \neq 1$. If $n=0$, the surface is isomorphic to $\mathbb{P}^{1} \times \mathbb{P}^{1}$ and, if $n=1$, the surface is not minimal since it is isomorphic to the blowup of $\mathbb{P}^{2}$ at one point. For all $n \geq 1$ there is a unique irreducible curve on $\mathbf{F}_{n}$ with negative self-intersection (equal to $-n$ ). It is defined by a section of the $\mathbb{P}^{1}$-bundle $\mathbf{F}_{n} \rightarrow \mathbb{P}^{1}$ corresponding to the surjection $\mathcal{O} \oplus \mathcal{O}(-n) \rightarrow \mathcal{O}(-n)([26], \S \mathrm{V} .2)$.

Proof of Proposition 2.2. Under assumption (i), the Riemann-Roch formula on a rational surface and Serre's Duality,

$$
h^{0}(D)+h^{0}\left(K_{X}-D\right)=h^{1}(D)+\frac{1}{2} D \cdot\left(D-K_{X}\right)+1
$$

and $K_{X}^{2}=0$ imply that $h^{0}\left(-K_{X}\right)>0$. Let $F_{0}$ be an element of the linear system $\left|-K_{X}\right|$.

We now prove $(\mathrm{i}) \Leftrightarrow($ ii). The exact sequence

$$
0 \rightarrow \mathcal{O}_{X} \rightarrow \mathcal{O}_{X}\left(n F_{0}\right) \rightarrow \mathcal{O}_{F_{0}}\left(n F_{0}\right) \rightarrow 0
$$

together with $h^{1}\left(X, \mathcal{O}_{X}\right)=0$, because $X$ is rational, show that

$$
h^{0}\left(\mathcal{O}_{X}\left(n F_{0}\right)\right)=1+h^{0}\left(\mathcal{O}_{F_{0}}\left(n F_{0}\right)\right) .
$$

Since $F_{0}$ is a nef divisor and $F_{0}^{2}=0$, the restriction of $\mathcal{O}_{X}\left(n F_{0}\right)$ to each irreducible component of $F_{0}$ is an invertible sheaf of degree zero. The curve $F_{0}$ is of arithmetic genus 1 , so we can apply the Riemann-Roch Theorem on $F_{0}$ (see [33], Lecture 11) to conclude that $h^{0}\left(\mathcal{O}_{F_{0}}\left(n F_{0}\right)\right)>0$ if and only if $\mathcal{O}_{F_{0}}\left(n F_{0}\right) \cong \mathcal{O}_{F_{0}}$ if and only if $h^{0}\left(\mathcal{O}_{F_{0}}\left(n F_{0}\right)\right)=1$.

This shows that the index $m$ can be characterized by the property

$$
m=\min \left\{n: h^{0}\left(\mathcal{O}_{X}\left(-n K_{X}\right)\right)=2\right\}=\min \left\{n: h^{0}\left(\mathcal{O}_{F_{0}}\left(n F_{0}\right)\right)>0\right\},
$$

and the equivalence (i) $\Leftrightarrow$ (ii) follows from this characterization.

(i) $\Rightarrow$ (iii) The pencil $\left|-m K_{X}\right|$ defines a morphism $f: X \rightarrow \mathbb{P}^{1}$ with general fiber of arithmetic genus 1 . The generic fiber $X_{\eta}$ is an irreducible curve of arithmetic genus 1 over the field $\mathbb{K}(\eta)$ of rational functions on the curve $\mathbb{P}^{1}$. Since $X$ is smooth,

\footnotetext{
${ }^{3}$ Here we adopt Grothendieck's definition of the projective bundle associated to a locally free sheaf.
} 
$X_{\eta}$ is a regular curve over $\mathbb{K}(\eta)$. It is known that it is smooth if $\operatorname{char}(\mathbb{K}) \neq 2,3$, so that in this case $f$ is an elliptic fibration (see [12, Proposition 5.5.1). If it is not smooth, then a general fiber of $f$ is an irreducible cuspidal curve, so that $f$ is a quasi-elliptic fibration. As explained above (just before Proposition 2.2) the fibration $f$ is relatively minimal.

Since $X$ is a rational nonminimal surface, there exists a (-1)-curve $E$ on $X$ satisfying $E \cdot K_{X}=-1$. This shows that $K_{X}$ is a primitive divisor class, i.e. $K_{X}$ is not a multiple of any other divisor class. If $m=1$, this implies that there are no multiple fibers. If $m>1$, this implies that the multiplicity $n$ of any multiple fiber $n D$ divides $m$. Since $\left|m F_{0}\right|$ is a multiple fiber of multiplicity $m$, the class of the divisor $\frac{m}{n} F_{0}-D$ is a torsion element in the Picard group of $X$. Since $X$ is a rational surface, this class must be trivial, and we conclude that $f$ has a unique multiple fiber, namely $m F_{0}$.

(iii) $\Rightarrow$ (iv) Since the fibration $f$ is relatively minimal, the canonical class is proportional to the class of the fibers of $f$ (see [1], $\S \mathrm{V} .12$ ); in particular, $K_{X}^{2}=0$ and $-K_{X}$ is nef.

Let $\pi: X \rightarrow Y$ be a birational morphism to a minimal ruled surface. Suppose that $Y$ is not isomorphic to $\mathbb{P}^{2}$; then $Y$ is isomorphic to a surface $\mathbf{F}_{n}, n \neq 1$. Let $E_{0}$ be the section of $Y$ with $E_{0}^{2}=-n$ and let $E$ be its proper transform on $X$. We have $E^{2} \leq-n$ and $E^{2}=-n$ if and only if $\pi$ is an isomorphism in an open neighborhood of $E_{0}$. Since $-K_{X}$ is nef, Lemma 2.1 shows that $n=0$ or $n=2$. Assume $n=2$. Then $\pi$ is an isomorphism over $E_{0}$; hence it factors through a birational morphism $\pi: X \rightarrow X_{1}$, where $X_{1}$ is the blowup of $\mathbf{F}_{2}$ at a point $x \notin E_{0}$. Let $X_{1} \rightarrow \mathbf{F}_{1}$ be the blowdown of the fiber of the ruling $\mathbf{F}_{2} \rightarrow \mathbb{P}^{1}$ passing through $x$. Then we obtain a birational morphism $X \rightarrow \mathbf{F}_{1} \rightarrow \mathbb{P}^{2}$. Assume now that $n=0$. The morphism factors through $X \rightarrow X_{2}$, where $X_{2}$ is the blowup of a point $y$ on $\mathbf{F}_{0}$. Then we compose $X \rightarrow X_{2}$ with the birational morphism $X_{2} \rightarrow \mathbb{P}^{2}$ which is given by the stereographic projection of $\mathbf{F}_{0}$ onto $\mathbb{P}^{2}$ from the point $y$. As a consequence, changing $Y$ and $\pi$, we can always assume that $Y=\mathbb{P}^{2}$.

Since $K_{X}^{2}=0$, the morphism $\pi: X \rightarrow \mathbb{P}^{2}$ is the blowup of 9 points $p_{1}, \ldots, p_{9}$, where some of them may be infinitely near. Since $-K_{X}$ is nef, any smooth rational curve has self-intersection $\geq-2$. This implies that the set of points $\left\{p_{1}, \ldots, p_{9}\right\}$ can be written in the form

$$
\left\{p_{1}^{(1)}, p_{1}^{(2)}, \ldots, p_{1}^{\left(a_{1}\right)} ; \ldots ; p_{k}^{(1)}, p_{k}^{(2)}, \ldots, p_{k}^{\left(a_{k}\right)}\right\},
$$

where the $p_{j}^{(1)}$ are points in $\mathbb{P}^{2}$, and $p_{j}^{(b+1)}$ is infinitely near, of the first order, to the previous point $p_{j}^{(b)}$ for $j=1, \ldots, k$ and $b=1, \ldots, a_{j}-1$. Equivalently, the exceptional curve

$$
E_{j}=\pi^{-1}\left(p_{j}^{(1)}\right)
$$

is a chain of $(-2)$-curves of length $\left(a_{j}-1\right)$ with one more $(-1)$-curve at the end of the chain.

The formula for the canonical class of the blowup of a nonsingular surface at a closed point shows that

$$
K_{X}=-3 e_{0}+e_{1}+\cdots+e_{9},
$$

where $e_{0}=c_{1}\left(\pi^{*}\left(\mathcal{O}_{\mathbb{P}^{2}}(1)\right)\right.$ and $e_{j}$ is the divisor class of $E_{j}, j=1, \ldots, 9$. This implies that

$$
\left|-m K_{X}\right|=\left|3 m e_{0}-m\left(e_{1}+\cdots+e_{9}\right)\right|
$$


hence the image of the pencil $\left|-m K_{X}\right|$ in the plane is the linear system of curves of degree $3 m$ with singular points of multiplicity $m$ at $p_{i}^{(1)}, 1 \leq j \leq k$.

(iv) $\Rightarrow$ (i) Let $X$ be the blowup of the base points of the pencil. The proper transform of the pencil on $X$ is the linear system $\left|3 m e_{0}-m\left(e_{1}+\cdots+e_{9}\right)\right|$. The formula for the canonical class on $X$ shows that this system is equal to $\left|-m K_{X}\right|$. Since the pencil is irreducible, $\left|-m K_{X}\right|$ is a pencil with no fixed component and no base point, so $X$ is a Halphen surface.

Remark 2.3. The proof of the proposition shows that the multiplicity $m$ of the multiple fiber $m F_{0}$ of the genus one fibration is equal to the order of $\mathcal{O}_{F_{0}}\left(F_{0}\right)$ in $\operatorname{Pic}\left(F_{0}\right)$. This property characterizes nonwild fibers of elliptic fibrations (see [12], Proposition 5.1.5). It is a consequence of the vanishing of $H^{1}\left(X, \mathcal{O}_{X}\right)$. It always holds if the multiplicity is prime to the characteristic.

2.2. Halphen pencils of index $m$. The following lemma is well known and its proof is left to the reader.

Lemma 2.4. Let $\phi: S^{\prime} \rightarrow S$ be the blowup of a point $x$ on a smooth projective surface $S$ and let $C^{\prime}$ be the proper transform of a curve passing through $x$ with multiplicity 1. Then $\mathcal{O}_{C^{\prime}}\left(C^{\prime}+E\right) \cong\left(\phi_{\mid C^{\prime}}\right)^{*} \mathcal{O}_{C}(C)$, where $E=\phi^{-1}(x)$ is the exceptional divisor.

In the plane $\mathbb{P}^{2}$, an irreducible pencil of elliptic curves of degree $3 m$ with 9 base points of multiplicity $m$ is called a Halphen pencil of index $m$. If $C_{0}$ is a cubic curve through the base points, then $C_{0}$ is the image of a curve $F_{0} \in\left|-K_{X}\right|$; such a curve is unique if $m>1$ and moves in the pencil if $m=1$.

The classification of fibers of genus 1 fibrations shows that $\mathcal{O}_{F_{0}}\left(F_{0}\right) \approx \mathcal{O}_{F_{0}}$ implies that $F_{0}$ is a reduced divisor of type $I_{m}$ in Kodaira's notation, unless char $(\mathbb{K})$ divides $m$ (see [12, Proposition 5.1.8). We further assume that $F_{0}$ is irreducible if $m>1$; this will be enough for our applications. Thus $F_{0}$ is a smooth or nodal curve, unless the characteristic of $\mathbb{K}$ divided $m$ in which case it could be a cuspidal curve. Under this assumption, the restriction of $\pi$ to $F_{0}$ is an isomorphism $F_{0} \cong C_{0}$; in particular, no base point $p_{i}^{(j)}$ is a singular point of $C_{0}$.

In the notation of Equation (3), consider the divisor class in $\operatorname{Pic}\left(C_{0}\right)$ given by

$$
\mathfrak{d}=3 \mathfrak{h}-a_{1} p_{1}^{(1)}-\cdots-a_{k} p_{k}^{(1)},
$$

where $\mathfrak{h}$ is the intersection of $C_{0}$ with a line in the plane. Since $\mathcal{O}_{C_{0}}\left(C_{0}\right) \cong \mathcal{O}_{C_{0}}(3 \mathfrak{h})$, Lemma 2.4 gives

$$
\mathcal{O}_{F_{0}}\left(F_{0}\right) \cong \mathcal{O}_{F_{0}}\left(3 e_{0}-e_{1}-\cdots-e_{9}\right) \cong\left(\left.\pi\right|_{F_{0}}\right)^{*}\left(\mathcal{O}_{C_{0}}(\mathfrak{d})\right) .
$$

This implies that $\mathcal{O}_{F_{0}}\left(F_{0}\right)$ is of order $m$ in $\operatorname{Pic}\left(F_{0}\right)$ if and only if $\mathfrak{d}$ is of order $m$ in $\operatorname{Pic}\left(C_{0}\right)$. If we choose the group law $\oplus$ on the set $C_{0}^{\#}$ of regular points of $C_{0}$ with a nonsingular inflection point $o$ as the zero point, then the latter condition is equivalent to

$$
a_{1} p_{1}^{(1)} \oplus \cdots \oplus a_{k} p_{k}^{(1)}=\epsilon_{m},
$$

where $\epsilon_{m}$ is a point of order $m$ in the group $\left(C_{0}^{\#}, \oplus\right)$.

This provides a way to construct Halphen pencils and the corresponding Halphen surfaces (under our assumptions that $F_{0}$ is irreducible). Start with an irreducible plane cubic $C_{0}$, and choose $k$ points $p_{1}^{(1)}, \ldots, p_{k}^{(1)}$ in $C_{0}^{\#}$ satisfying Equation (5) with 
$a_{1}+\cdots+a_{k}=9$. Then blow up the points $p_{1}^{(1)}, \ldots, p_{k}^{(1)}$ together with infinitely near points $p_{j}^{(i)}, i=2, \ldots, a_{j}$, to arrive at a rational surface $\pi: X \rightarrow \mathbb{P}^{2}$. Then $\left|-K_{X}\right|=\left|F_{0}\right|$, where $F_{0}$ is the proper transform of $C_{0}$. Since the $p_{i}^{(1)}$ satisfy Equation (5), $\mathcal{O}_{F_{0}}\left(F_{0}\right) \cong\left(\left.\pi\right|_{F_{0}}\right)^{*} \mathcal{O}_{C_{0}}\left(\epsilon_{m}\right)$ is of order $m$ in $\operatorname{Pic}\left(F_{0}\right)$. Since $F_{0}$ is irreducible, $\left|-m K_{X}\right|=\left|m F_{0}\right|$ is nef. Consequently, Proposition 2.2 shows that $X$ is a Halphen surface.

2.3. Unnodal Halphen surfaces. By Lemma 2.1, Halphen surfaces contain no $(-n)$-curves with $n \geq 3$. Recall that a Halphen surface is unnodal if it has no $(-2)$-curves. Since a $(-2)$-curve $R$ satisfies $R \cdot K_{X}=0$, it must be an irreducible component of a fiber of the genus 1 fibration $f: X \rightarrow \mathbb{P}^{1}$. Conversely, all reducible fibers of $f$ contain $(-2)$-curves, because $f$ is a relatively minimal elliptic fibration. Thus $X$ is unnodal if and only if all members of the pencil $\left|-m K_{X}\right|$ are irreducible.

In this case all the curves $E_{i}$ are $(-1)$-curves; in particular, there are no infinitely near points in the Halphen set. Also, in this case, the morphism $f: X \rightarrow \mathbb{P}^{1}$ is an elliptic fibration because any quasi-elliptic fibration on a rational surface has a reducible fiber whose irreducible components are $(-2)$-curves (this follows easily from [12, Proposition 5.1.6; see the proof of Theorem 5.6.3). The fibers of $f$ are irreducible curves of arithmetic genus 1. This shows that a Halphen surface is unnodal if and only if it arises from a Halphen pencil with irreducible members.

Proposition 2.5. Let $X$ be a Halphen surface of index $m$. Then $X$ is unnodal if and only if the following conditions are satisfied.

(i) There is no infinitely near point in the Halphen set $\mathcal{P}=\left\{p_{1}, \ldots, p_{9}\right\}$;

(ii) the divisor classes

$$
\begin{aligned}
& -d K_{X}+e_{i}-e_{j}, i \neq j, \quad 0 \leq 2 d \leq m, \\
& -d K_{X} \pm\left(e_{0}-e_{i}-e_{j}-e_{k}\right), i<j<k, 0 \leq 2(3 d \pm 1) \leq 3 m
\end{aligned}
$$

are not effective.

Remark 2.6. Since $K_{X}=-3 e_{0}+\sum e_{i}$, the two types of divisor classes in condition (ii) are equal to

$$
\begin{aligned}
& 3 d e_{0}-d\left(e_{1}+\cdots+e_{9}\right)+e_{i}-e_{j}, i \neq j, \\
& 3 d e_{0}-d\left(e_{1}+\cdots+e_{9}\right) \pm\left(e_{0}-e_{i}-e_{j}-e_{k}\right), i<j<k .
\end{aligned}
$$

Example 2.7. When $m=1$, the inequalities $2 d \leq m$ and $2(3 d \pm 1) \leq 3 m$ lead to $d=0$ and the conditions are respectively redundant with (i), or exclude triples of collinear points in the set $\left\{p_{1}, \ldots, p_{9}\right\}$.

When $m=2$, the inequality $2 d \leq m$ reads $d=0$ or 1 , and we have to exclude a cubic through 8 points with a double point at one of them. The inequality $2(3 d \pm 1) \leq 3 m$ gives rise to curves of degree 1 (for $d=0)$ and degree 2 (for $d=1$ ). We have to exclude triples of collinear points and sets of six points on a conic.

Proof of Proposition 2.5. Suppose the conditions are satisfied. Since no class $e_{i}-e_{j}$ is effective, the exceptional curves $E_{i}$ are $(-1)$-curves. Thus the morphism $\pi: X \rightarrow$ $\mathbb{P}^{2}$ is the blowup of 9 points, none of which is infinitely near another. This implies that $\pi$ does not contract any component of a member of $\left|-m K_{X}\right|$. Let $R$ be a $(-2)$-curve on $X$. It must be an irreducible component of a member of $\left|-m K_{X}\right|$; 
hence $\bar{R}=\pi(R)$ is an irreducible component of a curve of degree $3 \mathrm{~m}$. Taking a complementary component, we may assume that

$$
2 \operatorname{deg}(\bar{R}) \leq 3 m \text {. }
$$

The divisor class $r=[R]$ belongs to $K_{X}^{\perp}$ and satisfies $r^{2}=-2$. Since $\left(r+d K_{X}\right)^{2}=$ -2 , we can change $r$ into $r^{\prime}=r+d K_{X}$ in such a way that $r^{\prime}=d^{\prime} e_{0}-k_{1}^{\prime} e_{1}-\cdots-k_{9}^{\prime} e_{9}$ with $\left|d^{\prime}\right| \leq 1$. All such vectors $r^{\prime}$ can be listed:

$$
r^{\prime}=e_{i}-e_{j}, i \neq j, \quad \text { or } \quad r^{\prime}= \pm\left(e_{0}-e_{i}-e_{j}-e_{k}\right), i<j<k .
$$

Thus

$$
\begin{aligned}
& r=-d K_{X}+e_{i}-e_{j}, \quad \text { or } \\
& r=-d K_{X} \pm\left(e_{0}-e_{i}-e_{j}-e_{k}\right), i<j<k .
\end{aligned}
$$

Since $\operatorname{deg} \bar{R}=r \cdot e_{0}>0$ and $-K_{X} \cdot e_{0}=3$, the curve $\bar{R}$ is of degree $3 d$ in the first case, and of degree $3 d \pm 1$ in the second case. Thus, inequality (6) shows that $2 d \leq m$ (resp. $2(3 d \pm 1) \leq 3 m)$. From (ii), we deduce that $\bar{R}$ and $R$ do not exist, and that $X$ is general.

Conversely, if one of these divisor classes is effective, then $X$ contains a $(-2)$ curve. This proves the proposition.

Remark 2.8. Let $f: X \rightarrow \mathbb{P}^{1}$ be a Halphen elliptic surface of index $m>1$. The generic fiber $X_{\eta}$ is a genus one curve over the field $\mathbb{K}(\eta)$ which has no rational point over this field. The Jacobian variety $\operatorname{Jac}\left(X_{\eta}\right)$ of $X_{\eta}$ (equal to the connected component of the identity of the Picard scheme of $X_{\eta}$ over $\eta$ ) is an abelian variety of dimension 1 over $\mathbb{K}(\eta)$. Applying the theory of relative minimal models one can construct an elliptic surface $j: J \rightarrow \mathbb{P}^{1}$ with generic fibers $J_{\eta}$ isomorphic to $\operatorname{Jac}\left(X_{\eta}\right)$. It is called the Jacobian elliptic surface of $X \rightarrow \mathbb{P}^{1}$. In our case, this elliptic surface is a Halphen surface of index 1 . The curve $X_{\eta}$ is a torsor over $J_{\eta}$. Its class in the group of isomorphism classes of torsors over $J_{\eta}$ is uniquely determined by a choice of a closed point $y \in \mathbb{P}^{1}$ and an element $\alpha$ of order $m$ in $\operatorname{Pic}\left(J_{y}\right)$. The fiber $X_{y}=m F_{y}$ is the unique multiple fiber of $f$, the curves $F_{y}$ and $J_{y}$ can be canonically identified and the isomorphism class of $\mathcal{O}_{F_{y}}\left(F_{y}\right)$ coincides with $\alpha$. Since Halphen surfaces of index 1 are parameterized by an open subset of the Grassmannian $G(2,10)$ of pencils of plane cubic curves, their moduli space is an irreducible variety of dimension 8 . The construction of the Jacobian surface shows that the moduli space of Halphen surfaces of index $m>1$ is a fibration over the moduli space of Halphen surfaces of index 1 with one-dimensional fibers. It is expected to be an irreducible variety of dimension 9 .

Remark 2.9. It follows from Proposition 2.5 that unnodal Halphen sets of given index form a proper Zariski open subset in the set of all Halphen sets of this index. So, one can say that unnodal Halphen sets or the corresponding Halphen surfaces are general in the sense of moduli.

2.4. Automorphisms of a Halphen surface. We now discuss a result of Coble (see a modern proof in 23]) which describes the automorphism group of an unnodal Halphen surface $X$ and its image in the group $W_{X}$.

Let $X$ be a Halphen surface of index $m$. Since the group Aut $(X)$ preserves the canonical class $K_{X}$, it preserves the linear system $\left|-m K_{X}\right|$ and permutes the fibers of the Halphen fibration $f: X \rightarrow \mathbb{P}^{1}$. As explained in the Introduction, see Section 1.3, we identify $\operatorname{Pic}(X)$ with $\mathbb{Z}^{1,9}$ and $W_{X}$ with the Coxeter group $W_{9}$. The lattice 
$\mathbb{E}_{9} \cong K_{X}^{\perp}$ is isomorphic to the root lattice of affine type $E_{8}$; the radical of $\mathbb{E}_{9}$ is generated by the vector $k_{9}$ and the lattice $\mathbb{E}_{8} \cong \mathbb{E}_{9} / \mathbb{Z} k_{9}$ is isomorphic to the root lattice of finite type $E_{8}$. Consequently, the Weyl group $W_{9}$ is isomorphic to the affine Weyl group of type $E_{8}$ and fits in the extension

$$
0 \rightarrow \mathbb{E}_{8} \stackrel{\iota}{\longrightarrow} W_{9} \rightarrow W_{8} \rightarrow 1
$$

where $\iota: \mathbb{E}_{8} \rightarrow W_{9}$ is defined by the formula

$$
\iota(w)(v)=v+\left(v, k_{9}\right) w-\left((w, v)+\frac{1}{2}\left(v, k_{9}\right)(w, w)\right) k_{9},
$$

and $W_{8}$ is a finite group of order $2^{7} \cdot 3^{3} \cdot 5 \cdot 8 ! 4$

The following theorem shows that the size of $\operatorname{Aut}(X)$ depends on the existence of reducible fibers for $f$ (see [23] for a more precise statement). We identify the lattice $\mathbb{E}_{8}$ with $K_{X}^{\perp} / \mathbb{Z} K_{X}$ and the map $\iota$ with the homomorphism $K_{X}^{\perp} / \mathbb{Z} K_{X} \rightarrow W_{X}$ defined by $\iota(D)(A)=A-(A \cdot D) K_{X}$.

Theorem 2.10. Let $X$ be a Halphen surface of index $m$. If $X$ is unnodal, then Aut $(X)^{*}$ contains a subgroup $G$ whose image in $W_{9}$ is equal to $\iota\left(m \mathbb{E}_{8}\right) \subset \iota\left(\mathbb{E}_{8}\right)$; in particular, an unnodal Halphen set is Cremona special. If $X$ is not unnodal, then the index of $\operatorname{Aut}(X)^{*}$ in $W_{X}$ is infinite, and thus $X$ is not Cremona special.

Proof. Let $f: X \rightarrow \mathbb{P}^{1}$ be the elliptic fibration defined by $\left|-m K_{X}\right|$ and $X_{\eta}$ be its generic fiber. Assume $X$ is unnodal. Then $X_{\eta}$ is a smooth elliptic curve over the field $\mathbb{K}(\eta)$ of rational functions on $\mathbb{P}^{1}$ (see the beginning of Section 2.3). The closure in $X$ of a closed point $\zeta \in X_{\eta}$ of degree $d(\zeta)=[\mathbb{K}(\zeta): \mathbb{K}(\eta)]$ is an irreducible curve $\bar{\zeta}$ such that the restriction of $\pi$ to the curve is a finite cover of degree $d(\zeta)$. Conversely, any irreducible curve $C$ of relative degree $d$ (a $d$-multi-section) intersects $X_{\eta}$ at a closed point of degree $d$. In the case of an unnodal Halphen surface, any exceptional curve $E_{i}$ is of relative degree $m$ and the degree of any closed point on $X_{\eta}$ is a multiple of $m$. In particular, $X_{\eta}$ has a structure of an abelian variety only if $m=1$.

Let $J_{\eta}=\mathbf{P i c}_{X_{\eta} / \eta}^{0}$ be the Jacobian variety of $X_{\eta}$. Now it is an abelian curve which acts on $X_{\eta} \cong \mathbf{P i c}_{X_{\eta} / \eta}^{1}$ by translations. In particular, $J_{\eta}(\eta)$ acts by $\eta$ automorphisms on $X_{\eta}$. Explicitly, the action is defined by the formula

$$
t_{\mathfrak{a}}(x)=x^{\prime} \sim x+\mathfrak{a}, \quad \mathfrak{a} \in J_{\eta}(\eta),
$$

where $x$ is a closed point of $X_{\eta}$ over the algebraic closure of $\mathbb{K}(\eta)$. Since $\mathfrak{a}$ is defined over $\mathbb{K}(\eta)$, this guarantees that the action is defined over $\eta$.

It follows from the theory of relative minimal models that the action of $J_{\eta}(\eta)$ on $X_{\eta}$ extends to a biregular action of $X / \mathbb{P}^{1}$. Thus we obtain an injective morphism

$$
J_{\eta}(\eta) \hookrightarrow \operatorname{Aut}(X) .
$$

By taking the closure of a divisor on $X_{\eta}$, we have a natural surjective restriction homomorphism

$$
\operatorname{tr}: K_{X}^{\perp} \rightarrow J_{\eta}(\eta)
$$

Its kernel is equal to the subgroup $\operatorname{Pic}_{\text {fib }}(X)$ of $\operatorname{Pic}(X)$ generated by irreducible components of fibers. If $X$ is unnodal, $\operatorname{Pic}_{\mathrm{fib}}(X)=\mathbb{Z} K_{X}$; hence $J_{\eta}(\eta) \cong \mathbb{Z}^{8}$. This proves that $\operatorname{Aut}(X)$ contains a subgroup isomorphic to $\mathbb{Z}^{8}$.

It remains to find out how this abelian group $J_{\eta}(\eta)$ acts on $\operatorname{Pic}(X)$. Any effective divisor $D$ on $X$ is a sum of irreducible components of fibers and the rest, which

\footnotetext{
${ }^{4}$ It is an exercise to check that $\iota\left(w+w^{\prime}\right)=\iota(w) \circ \iota\left(w^{\prime}\right)$.
} 
we call the horizontal part. The restriction of the fibration $f: X \rightarrow \mathbb{P}^{1}$ to the horizontal part is a finite cover of degree equal to

$$
\operatorname{deg}_{f}(D)=-m D \cdot K_{X}
$$

We say that an effective divisor is separable if its horizontal part is a separable cover of $\mathbb{P}^{1}$. If $\operatorname{char}(\mathbb{K})$ does not divide $m$, any effective divisor is separable.

For any divisor class $D$ on $X$ let $\operatorname{tr}(D)$ be its scheme-theoretical intersection with the generic fiber $X_{\eta}$. If $D$ is separable and irreducible, then $\operatorname{tr}(D)$ is a closed point of $X_{\eta}$ which is equal to the sum of $\operatorname{deg}_{f}(D)$ closed points over the algebraic closure of $\mathbb{K}(\eta)$. Thus $t_{\mathfrak{a}}(\operatorname{tr}(D))=\operatorname{tr}(D)+\operatorname{deg}_{f}(D) \mathfrak{a}$. Let $A$ be an element of $K_{X}^{\perp}$ such that $\operatorname{tr}(A)=\mathfrak{a}$. Then

$$
t_{\mathfrak{a}}(D) \sim D-m\left(D \cdot K_{X}\right) A \bmod \operatorname{Pic}_{\mathrm{fib}}(X) .
$$

At this point, we assume that $X$ is unnodal, so that

$$
t_{\mathfrak{a}}(D) \sim D-m\left(D \cdot K_{X}\right) A+\lambda K_{X}
$$

for some integer $\lambda$. Intersecting both sides with $D$ and $D^{\prime}=t_{\mathfrak{a}}(D)$, and using that $D^{2}=D^{\prime 2}$, we obtain $\lambda=\frac{m}{2}\left(D \cdot A+D^{\prime} \cdot A\right)$. Intersecting both sides with $A$, we obtain $D^{\prime} \cdot A=D \cdot A-m(K \cdot D) A^{2}$. Combining the two formulas, we get

$$
t_{\mathfrak{a}}(D) \sim D-m\left(D \cdot K_{X}\right) A+\left[m(D \cdot A)-\frac{m^{2}}{2}\left(D \cdot K_{X}\right) A^{2}\right] K_{X}
$$

(cf. 23, Proposition 9, where the sign in front of $m^{2}$ must be changed). This should be compared with formula (9). Since $\operatorname{Pic}(X)$ is generated by separable effective divisors (e.g. by the divisor classes $\left.e_{0}, \ldots, e_{9}\right)$, this determines the action of $J_{\eta}(\eta)$ on $\operatorname{Pic}(X)$. Restricting to $K_{X}^{\perp}$, we obtain

$$
t_{\mathfrak{a}}(D) \sim D+m(D \cdot A) K_{X} .
$$

So, the image of $K_{X}^{\perp} / \mathbb{Z} K_{X}$ in $\operatorname{Aut}(X)$ acts on $K_{X}^{\perp}$ as the subgroup $\iota\left(m K_{X}^{\perp}\right)$ of $W_{X}$. This proves the first assertion of the proposition.

Assume now that $X$ is not unnodal. The set of $(-2)$-curves on $X$ is not empty and coincides with the set of irreducible components of reducible fibers of $f$. The group $\operatorname{Aut}(X)$ permutes the elements of this finite set. Thus, a finite index subgroup of $\operatorname{Aut}(X)$ fixes all the divisor classes of these $(-2)$-curves. The subgroup of all elements of $\mathbb{E}_{8} \subset W_{9}$ that fix such a class has infinite index in $\mathbb{E}_{8}$; more precisely, the rank of this free abelian group is at most $8-k$, where $k$ is the dimension of the subspace which is spanned by classes of $(-2)$-curves. This implies that $\operatorname{Aut}(X)$ has infinite index in $W_{9}$, and therefore that $X$ is not Cremona special.

Remark 2.11. Consider the variety $\widetilde{\operatorname{Halp}}(m)^{\text {gen }} \subset\left(\mathbb{P}^{2}\right)^{9}$ of ordered unnodal Halphen sets $\left(p_{1}, \ldots, p_{9}\right)$. It follows from the Hilbert-Mumford numerical criterion of stability that the GIT quotient $\widetilde{\mathrm{Halp}}^{\mathrm{gen}} / / \mathrm{SL}(3)$ exists and parameterizes the orbits of unnodal Halphen ordered sets of index $m$. The group $W_{9}$ acts on this space regularly by means of the Coble action (see [20]). Since two Halphen surfaces are isomorphic if and only if the corresponding Halphen sets are projectively equivalent, we obtain that the subgroup $m \mathbb{E}_{8}$ acts trivially on this variety; hence the quotient group $G=W_{9} / \mathbb{E}_{8} \cong\left(\mathbb{E}_{8} / m \mathbb{E}_{8}\right) \rtimes W_{8}$ acts on the orbit space.

Since all automorphisms of a Halphen surface preserve the elliptic pencil, we have a natural homomorphism $\rho: \operatorname{Aut}(X) \rightarrow \operatorname{Aut}\left(\mathbb{P}^{1}\right)$ whose image is a finite subgroup preserving the set of points corresponding to singular nonmultiple fibers. If $m>1$, 
it must also fix the multiple fiber, so the group is a cyclic group which has orbits of cardinality $\leq 12$. The kernel of $\rho$ is a subgroup of $\operatorname{Aut}\left(X_{\eta}\right)$ which is a finite extension of $J_{\eta}$ by a cyclic group of order dividing 24 (6 if $\operatorname{char}(\mathbb{K}) \neq 2,3$ ). If $m \leq 2$ the group of order 2 is always present. It corresponds to the automorphism $x \mapsto-x$ of the generic fiber if $m=1$ and the double cover $X_{\eta} \rightarrow \mathbb{P}_{\eta}^{1}$ given by a 2 -section if $m=2$. For $X$ general enough, we have $\operatorname{Aut}(X) \cong \mathbb{Z}^{8} \rtimes(\mathbb{Z} / 2 \mathbb{Z})$ if $m \leq 2$ and $\operatorname{Aut}(X) \cong \mathbb{Z}^{8}$ otherwise. The image of the generator of $(\mathbb{Z} / 2 \mathbb{Z})$ in $\operatorname{Aut}(X)^{*}$ is equal to an element of $W_{9}$ which is mapped to the center of the group $W_{8}=W_{9} / \iota\left(\mathbb{E}_{8}\right)$.

\section{Coble surfaces}

The construction of Cremona special point sets with 10 points is due to Coble (see [9], 10]). A Coble surface is a rational smooth surface $X$ such that the linear system $\left|-K_{X}\right|$ is empty, but $\left|-2 K_{X}\right|$ is not. The classification of such surfaces can be found in 21. In what follows, we only need the special case, where we additionally assume that $K_{X}^{2}=-1$ and $\left|-2 K_{X}\right|$ consists of an irreducible curve $C$. So, in this paper, a Coble surface is always assumed to be such a surface. A Coble set is a point set $\mathcal{P}$ such that the blowup of $\mathcal{P}$ is a Coble surface.

In this section, we study Coble surfaces and show that unnodal Coble surfaces are Cremona special. We provide a proof, which works in any characteristic. Most arguments and constructions of this section are used in Section 5, where we prove the Main Theorem.

3.1. From Coble to Halphen surfaces. Let $X$ be a Coble surface, and $C$ be an irreducible curve in the linear system $\left|-2 K_{X}\right|$. By definition, the arithmetic genus $p_{a}(C)$ satisfies

$$
p_{a}(C)=1+\frac{1}{2}\left(C^{2}+C \cdot K_{X}\right)=1+K_{X}^{2}=0 .
$$

Thus $C$ is a smooth rational curve with self-intersection $C^{2}=4 K_{X}^{2}=-4$.

Proposition 3.1. Let $X$ be a Coble surface and $\pi_{E}: X \rightarrow Y$ be the blowdown of a (-1)-curve E. Then

- $Y$ is a Halphen surface of index 2 ;

- $C$ is the proper transform of the fiber $F$ containing $y_{0}=\pi_{E}(E)$;

- the fiber $F$ is irreducible, and $y_{0}$ is its unique singular point.

Conversely, the blowup of a singular point of an irreducible nonmultiple fiber of a Halphen surface of index 2 is a Coble surface.

Proof. Since $K_{X}^{2}=-1$, we get $K_{Y}^{2}=0$. Let $y_{0}$ be the image of $E$ by $\pi_{E}$. Let $F$ be the image of $C$; since $C$ is irreducible, so is $F$. Since $C \cdot E=-2 K_{X} \cdot E=2$, the curve $F$ is singular at $y_{0}$. From $\pi_{E}^{*}(F)=C+2 E$, we deduce that $F$ is an irreducible curve in the linear system $\left|-2 K_{Y}\right|$; in particular, $F^{2}=0$ and $-K_{Y}$ is nef.

By Riemann-Roch, $h^{0}\left(-K_{Y}\right)>0$. If $h^{0}\left(-K_{Y}\right)>1$, we can find a curve $G$ from $\left|-K_{Y}\right|$ passing through $y_{0}$, and thus $\pi^{*}(G)-E$ is effective and $\left|-K_{X}\right| \neq \emptyset$, contradicting the definition of a Coble surface. Thus $h^{0}\left(-K_{Y}\right)=1$ and the unique effective divisor $F_{0}$ in $\left|-K_{Y}\right|$ does not pass through $y_{0}$. This implies that $\left|-2 K_{Y}\right|$ contains two linearly independent divisors, namely $2 F_{0}$ and $F$; hence $h^{0}\left(-2 K_{Y}\right) \geq$ 2. Since $K_{Y}^{2}=0,2 F_{0}$ and $F$ are disjoint, and the linear system $\left|-2 K_{Y}\right|$ has no 
fixed components; since it contains the reduced and irreducible curve $F$, this pencil is irreducible. Thus $Y$ is a Halphen surface of index 2.

Conversely, let $X$ be obtained from a Halphen surface $Y$ of index 2 as indicated in the assertion of the proposition. Since the irreducible fiber $F$ belongs to $\left|-2 K_{Y}\right|$ and its singular point is blown up, the linear system $\left|-2 K_{X}\right|$ is not empty and contains the proper transform of $F$, which is a smooth rational curve. Moreover, $\left|-K_{X}\right|$ is empty because $\left|-K_{Y}\right|$ consists of the unique multiple fiber $F_{0}$ of $Y$ and $F_{0}$ does not pass through the point which we blow up. So, $X$ is a Coble surface.

Let $\pi: X \rightarrow \mathbb{P}^{2}$ be the composition of $\pi_{E}: X \rightarrow Y$ and the blow-down morphism $\pi^{\prime}: Y \rightarrow \mathbb{P}^{2}$ which is described in Proposition 2.2. The image $F$ of $C$ in $Y$ belongs to $\left|-2 K_{Y}\right|=\left|6 e_{0}-2\left(e_{1}+\cdots+e_{9}\right)\right|$; hence its image in the plane is an irreducible plane curve of degree 6 with 10 singular points $p_{1}, \ldots, p_{10}$, maybe with some infinitely near points. This set $\left\{p_{1}, \ldots, p_{10}\right\}$ is a Coble set of 10 points. It contains a Halphen set of index 2 ; the remaining tenth point corresponds to the singular point of $F$. Conversely, starting from a set of 10 singular points $p_{1}, \ldots, p_{10}$ of an irreducible curve of degree 6 , we choose a point $p_{i}$ such that no other point is infinitely near it. The remaining set of 9 points is a Halphen point set of index 2 .

3.2. Unnodal Coble surfaces. By Lemma 2.1, a Coble surface $X$ has no $(-n)$ curves with $n \geq 3$ except the unique curve in $\left|-2 K_{X}\right|$ (with self-intersection -4 ). If $X$ is unnodal and $\pi_{E}: X \rightarrow Y$ is the blow-down map of a $(-1)$-curve $E$, then $Y$ is an unnodal Halphen surface of index 2. Otherwise the pre-image, in $X$, of a component of a reducible fiber would define a $(-2)$-curve or a $(-3)$ curve if $\pi_{E}(E)$ is a singular point of a reducible fiber of the elliptic pencil on $Y$. However, the converse is not generally true; one needs to impose more conditions on the point set to ensure that $X$ is unnodal.

Theorem 3.2. A Coble surface is unnodal if and only if it is obtained from a Coble set $\left\{p_{1}, \ldots, p_{10}\right\}$ satisfying the following 496 conditions:

(i) no points among the ten points are infinitely near;

(ii) no three points are collinear;

(iii) no six points lie on a conic;

(iv) no plane cubic passes through 8 points with one of them being a singular point of the cubic;

(v) no plane quartic curve passes through the 10 points with one of them being a triple point.

This result is due to A. Coble [9], (10). A modern proof was sketched in [11, Remark 4.7. We supply the full details here.

Proof of Theorem 3.2. It is known (and is easy to check) that the lattice $\mathbb{E}_{10} \cong K_{X}^{\perp}$ is a unimodular even lattice of signature $(1,9)$, hence isomorphic to the orthogonal sum $\mathbb{E}_{8} \perp \mathbb{H}$, where $\mathbb{H}$ is the hyperbolic plane defined by the matrix $\left(\begin{array}{ll}0 & 1 \\ 1 & 0\end{array}\right)$. Explicitly, the $\mathbb{E}_{8}$-part is generated by the vectors $\boldsymbol{\alpha}_{i}, i=0, \ldots, 7$. The sublattice generated by $\mathbb{E}_{8}$ and $\boldsymbol{\alpha}_{8}$ is isomorphic to $\mathbb{E}_{9}$. The radical of this copy of $\mathbb{E}_{9}$ and the vector $\boldsymbol{\alpha}_{9}$ generate the $\mathbb{H}$-part. Suppose conditions (i)-(v) are satisfied. Since there are no infinitely near points, $X$ is obtained from $\mathbb{P}^{2}$ by blowing up 10 distinct points $p_{i}$. We denote by $E_{i}$ the corresponding 10 exceptional divisors, and denote by $\left(e_{0}, e_{1}, \ldots, e_{10}\right)$ the geometric basis of $\operatorname{Pic}(X)$ (see Section 1.3). 
Let

$$
f_{i}=3 e_{0}-\left(e_{1}+\cdots+e_{10}\right)+e_{i}, i=1, \ldots, 10 .
$$

We have $f_{i} \cdot f_{j}=1-\delta_{i j}$. Let $Y_{i}$ be the Halphen surface of index 2 obtained from $X$ by blowing down the curve $E_{i}$. Then $f_{i}$ is the divisor class of the proper transform of the half-fiber of the elliptic fibration on $Y_{i}$. By Proposition 2.5. the first four conditions guarantee that the Halphen surfaces $Y_{i}$ are unnodal. The pencil $\left|2 f_{i}\right|$ is equal to the pre-image of the elliptic pencil on $Y_{i}$; it contains only one reducible fiber, namely $C+2 E_{i}$.

We have

$$
f_{1}+\cdots+f_{10}=30 e_{0}-9\left(e_{1}+\cdots+e_{10}\right)=3 \Delta
$$

with

$$
\Delta=10 e_{0}-3\left(e_{1}+\cdots+e_{10}\right)=-3 K_{X}+e_{0}, \quad \Delta^{2}=10, \quad \Delta \cdot K_{X}=0 .
$$

The linear system $|\Delta|$ is the proper transform of the linear system of plane curves of degree 10 passing through the points $p_{i}$ with multiplicities $\geq 3$. By counting constants, or applying Riemann-Roch, $\operatorname{dim}|\Delta| \geq 5$. Since the divisor classes $f_{i}$ are represented by irreducible curves, the divisor class $\Delta$ is nef and big. An irreducible curve $R$ on $X$ with $R \cdot \Delta=0$ satisfies $R \cdot f_{i}=0$, for all $i=1, \ldots, 10$, hence must coincide with the curve $C$. Thus $|\Delta|$ defines a morphism

$$
\phi: X \rightarrow \mathbb{P}^{\operatorname{dim}|\Delta|}
$$

that contracts $C$ onto a point $z$.

Consider the restriction of the linear system $|\Delta|$ to a general member $F_{i}$ of $\left|2 f_{i}\right|$. It is a linear series of degree 6 . Since

$$
\Delta-F_{i} \sim \Delta-2 f_{i}=4 e_{0}-\left(e_{1}+\cdots+e_{10}\right)-2 e_{i},
$$

by condition (v), this divisor class cannot be effective. This shows that the restriction of $\phi$ to $F_{i}$ is given by a complete linear system of degree 6 . Thus, $\phi_{\mid F_{i}}$ is an isomorphism onto a normal elliptic curve of degree 6 spanning $\mathbb{P}^{\operatorname{dim}|\Delta|}, \operatorname{dim}|\Delta|=5$ and $\phi$ is a birational isomorphism onto a surface of degree 10. This surface has a quotient singularity of type $\frac{1}{4}(1,1)$ at $z=\phi(C)$.

The remaining arguments follow the proof of Theorem 4.4 from [11, almost verbatim. Let $R$ be a $(-2)$-curve and $m_{0} e_{0}-m_{1} e_{1}-\cdots-m_{10} e_{10}$ be its divisor class. We have $m_{0}=R \cdot e_{0} \geq 0$ and $m_{i}=R \cdot E_{i} \geq 0$. Since $\Delta=-3 K_{X}+e_{0}$, we have $\Delta \cdot R=e_{0} \cdot R=m_{0}$. Suppose first that $m_{0} \leq 4$. Then listing all solutions of the diophantine equations

$$
\begin{aligned}
3 m_{0} & =m_{1}+\cdots+m_{10}, \\
m_{0}^{2}+2 & =m_{1}^{2}+\cdots+m_{10}^{2}
\end{aligned}
$$

with $m_{0} \leq 4$, we find that $R$ is contained in an exceptional curve coming from an infinitely near point, or is equal to the proper transform of one of the curves corresponding to conditions $(i i)$ to $(v)$, or belongs to the class $4 e_{0}-\left(e_{1}+\cdots+e_{10}\right)-$ $e_{i}-e_{j}-e_{k}+e_{s}$ with four distinct indices $i, j, k, s$. All cases except the last one are prohibited by the assumptions of the theorem. In the last case, the curve $R$ does not intersect the exceptional curve $E_{s}$; hence $R$ is coming from a $(-2)$-curve on the Halphen surface $Y_{s}$ obtained by blowing down $E_{s}$. It follows from Proposition 2.5 that in this case there exists a conic passing through the six points $p_{a}$ with $a \neq i, j, k, s$. This is prohibited by condition (iii). Thus, there is no (-2)-curve $R$ with $m_{0} \leq 4$. 
So, assume now that $m_{0}>4$ for any $(-2)$-curve $R$. Repeating the argument of the proof of Theorem 4.4 from [11], $R \cdot w(\Delta)>4$ for all $w \in W_{X}$. Taking $w$ to be the reflection with respect to $R$, we obtain $R \cdot w(\Delta)=w(R) \cdot \Delta=-R \cdot \Delta<0$, a contradiction.

Remark 3.3. Theorem 3.2 shows that unnodal Coble sets form a Zariski open subset in the set of all Coble sets. In particular, an unnodal Coble surface is a general Coble surface in the sense of moduli.

3.3. Effective divisors on unnodal Coble and Halphen surfaces. Recall that an element of $\mathbb{E}_{n}$ of norm -2 is called a root. The lattice is spanned by (simple) roots $\boldsymbol{\alpha}_{i}$ (cf. Section 1.2). A real root is a root which belongs to the $W_{n}$-orbit of one (or any) of these simple roots. It is known that all roots are real if and only if $n \leq 10$ (see, for example, [20], Remark 5, p. 79). Let $X$ be a rational surface obtained by blowing up a point set $\mathcal{P}$ with $|\mathcal{P}|=n$. Using an isomorphism $\mathbb{E}_{n} \rightarrow K_{X}^{\perp}$ defined by a choice of a geometric basis on $X$, we can transfer these definitions to elements of the lattice $K_{X}^{\perp}$; since $W_{X}$ acts transitively on the set of geometric bases, the definitions do not depend on the choice of a geometric basis.

We say that a root $\alpha$ in $K_{X}^{\perp}$ is effective if it can be represented by the divisor class of an effective divisor. Clearly, the divisor class of a (-2)-curve is an example of an effective root.

Later on we shall use the following result due to M. Nagata 35] which applies to Coble and Halphen surfaces. For the sake of completeness, we include a proof.

Lemma 3.4. Let $X \rightarrow \mathbb{P}^{2}$ be the blowup of $n \geq 9$ points such that $\left|-m K_{X}\right|$ contains an irreducible curve for some $m>0$. Suppose that $X$ has no $(-2)$-curves. Then,

(0) for any effective divisor $D$ and any $w \in W_{X}$, the class $w([D])$ is effective;

(i) for any $(-1)$-curve $E$ and any $w \in W_{X}$, the linear system $|w(E)|$ consists of a unique $(-1)$-curve;

(ii) for any simple root $\alpha_{i}$ and any $w \in W_{X}$, the linear system $\left|w\left(\alpha_{i}\right)\right|$ is empty;

(iii) for any primitive isotropic effective divisor class $f$ and any $w \in W_{X}$, the divisor class $w(f)$ is a primitive isotropic effective divisor class.

Proof. Let $C$ be an element of $\left|-m K_{X}\right|$. For any irreducible curve $Z \neq C$, we have $Z \cdot C=-m Z \cdot K_{X} \geq 0$. By the adjunction formula, $Z^{2} \geq-2$; since $X$ does not contain $(-2)$-curves, all irreducible curves $Z \neq C$ satisfy $Z^{2} \geq-1$. Moreover, $Z^{2}=-1$ implies that $Z$ is a $(-1)$-curve.

Let $Z$ be a curve with $Z^{2} \geq-1$ and let $d e_{0}-d_{1} e_{1}-\cdots-d_{n} e_{n}$ be its divisor class. We have $Z^{2}=d^{2}-d_{1}^{2}-\cdots-d_{n}^{2} \geq-1$, and $d=[Z] \cdot e_{0}>0$ unless $Z$ is one of the exceptional curves $E_{i}$. We claim that, for any $w \in W_{X}$,

$$
w([Z]) \cdot e_{0}>0, \quad \text { unless } w([Z])=e_{i} \text { for some } i>0 .
$$

We use induction on the length $l(w)$ of $w$ as a word in simple reflections. Write $w=s_{i} w^{\prime}$ with $l\left(w^{\prime}\right)<l(w)$, where $s_{i}$ is the reflection given by the simple root $\alpha_{i}$ (see $₫ 1.3)$. By induction $w^{\prime}([Z]) \cdot e_{0}>0$ or $w^{\prime}([C])=e_{j}$ for some $j>0$. In the latter case, $s_{i}\left(e_{j}\right)$ is either equal to $e_{k}$ for some index $k$ or to $e_{0}-e_{a}-e_{b}$ for some indices $a \neq b>0$; so the assertion is true in the latter case. In the first case, the claim is obvious for $\alpha_{i} \neq \alpha_{0}$; so we assume $\alpha_{i}=\alpha_{0}$. Let $w^{\prime}([Z])=d e_{0}-d_{1} e_{1}-\cdots-d_{n} e_{n}$ with $d \geq 1$. Then $w([Z])=w^{\prime}([Z])+\left(w^{\prime}([Z]) \cdot \alpha_{0}\right) \alpha_{0}$ implies that $w([Z]) \cdot e_{0}=$ 
$2 d-d_{1}-d_{2}-d_{3}$. Assume it is negative, i.e. $1 \leq d<\left(d_{1}+d_{2}+d_{3}\right) / 2$. Then

$$
\begin{gathered}
-1 \leq d^{2}-d_{1}^{2}-\cdots-d_{n}^{2}<\frac{1}{4}\left(d_{1}+d_{2}+d_{3}\right)^{2}-d_{1}^{2}-\cdots-d_{n}^{2} \\
<\frac{3}{4}\left(d_{1}^{2}+d_{2}^{2}+d_{3}^{2}\right)-d_{1}^{2}-\cdots-d_{n}^{2} .
\end{gathered}
$$

This gives

$$
-4<-d_{1}^{2}-d_{2}^{2}-d_{3}^{2}-4\left(d_{4}^{2}-\cdots-d_{n}^{2}\right)
$$

hence $d_{4}=\cdots=d_{n}=0$ and $d_{1}, d_{2}, d_{3} \leq 1$. Since $2 \leq 2 d<d_{1}+d_{2}+d_{3}$ we obtain

$$
w^{\prime}([Z])=e_{0}-e_{1}-e_{2}-e_{3}=\alpha_{0} .
$$

This contradicts $Z^{2} \geq-1$ and proves the claim.

(0) Let $[D]$ be the class of an irreducible curve, with $[D] \notin \mathbb{Q}[C]$. For all $w \in W_{X}$, we get $w([D]) \cdot K_{X}=[D] \cdot w^{-1}\left(K_{X}\right)=D \cdot K_{X}=-\frac{1}{m} D \cdot C \leq 0$; moreover, $D^{2} \geq-1$. By Riemann-Roch, $w([D])$ or $\left[K_{X}-w(D)\right]$ is effective. Intersecting $\left[K_{X}-w(D)\right]$ with $e_{0}$, and using (12), we see that $\left[K_{X}-w(D)\right]$ is not effective; hence $w([D])$ is effective.

Now let $D$ be any effective divisor. Write $D$ as a sum of irreducible components

$$
D=s C+\sum_{j=1}^{k} b_{j} Z_{j},
$$

where $s \geq 0, b_{j}>0$, and $Z_{j}$ is an irreducible curve with $\left[Z_{j}\right] \notin \mathbb{Q}[C]$ for all $1 \leq j \leq$ $k$. Then all classes $w\left(\left[Z_{j}\right]\right)$ are effective. Since $w([D])=s[C]+\sum_{j} b_{j} w\left(\left[Z_{j}\right]\right)$, the class $w([D])$ is effective.

(i) Let $E$ be a $(-1)$-curve which we identify with its divisor class. As we saw above, $w(E)$ is effective. Write an effective representative of $w(E)$ as a sum of irreducible components. Since $w(E)^{2}=-1<0$, some of the components must have negative self-intersection. Thus we can write

$$
w(E) \sim s C+a_{1} G_{1}+\cdots+a_{k} G_{k}+Z,
$$

where $G_{1}, \ldots, G_{k}$ are $(-1)$-curves, $Z$ is a sum of irreducible curves $Z_{j}$ with $Z_{j}^{2} \geq 0$, and at least one of the coefficients $s, a_{1}, \ldots, a_{k}$ is positive. Applying $w^{-1}$ we get

$$
E \sim s C+a_{1} w^{-1}\left(G_{1}\right)+\cdots+a_{k} w^{-1}\left(G_{k}\right)+w^{-1}(Z) .
$$

From Property (0), we know that the classes $w^{-1}\left(G_{i}\right), 1 \leq i \leq k$, and $w^{-1}(Z)$ are effective. Since $E$ is a $(-1)$-curve, we have $|E|=\{E\}$. Also $E \neq C$ since $E^{2}=E \cdot K_{X}=-1$ but $C^{2}=m^{2} K_{X}^{2}$ and $C \cdot K_{X}=-m K_{X}^{2}$ cannot be both equal to -1. This implies that $s=0, Z=0, k=1$ and $E=w^{-1}\left(G_{1}\right)$; thus $|w(E)|=\left\{G_{1}\right\}$ and Property (i) is proved.

(ii) A simple root $\alpha_{i}=e_{i}-e_{i+1}$ is effective if and only if there are infinitely near points, which is excluded since we know that $X$ has no $(-n)$-curves with $n \geq 2$. The simple root $\alpha_{0}=e_{0}-e_{1}-e_{2}-e_{3}$ is effective if and only if the points $p_{1}, p_{2}$, $p_{3}$ are collinear. The proper transform of the corresponding line is a $(-2)$-curve; hence this root is not effective. Suppose $w\left(\alpha_{i}\right)$ is effective for some index $i \geq 0$ and some element $w$ in $W_{X}$. Write an effective representative as in Equation (13):

$$
w(\alpha) \sim s C+a_{1} G_{1}+\cdots+a_{k} G_{k}+Z .
$$

Applying $w^{-1}$, and using the same argument as in (i), we get a contradiction. 
(iii) By $(0)$ we know that $f^{\prime}=w(f)$ is effective. This class is isotropic because $w$ preserves the intersection form. It is primitive because otherwise $f=w^{-1}\left(f^{\prime}\right)$ is not primitive.

3.4. Automorphisms of unnodal Coble surfaces. Here we provide a proof, valid in any characteristic, of the following theorem which is implicitly contained in [9] (expressed in terms of projective orbits of point sets; see 91.5); several steps of the proof are used in Section 5.

Theorem 3.5. Let $X$ be an unnodal Coble surface. Then $\operatorname{Aut}(X)^{*}$ contains the subgroup of $W_{X}$ which is isomorphic to

$$
W_{10}(2):=\left\{w \in W_{10}: w(v)-v \in 2 \mathbb{E}_{10} \quad \text { for all } v \in \operatorname{Pic}(X)\right\}
$$

under the natural identification of $W_{X}$ with $W_{10}$.

Remark 3.6. The subgroup $W_{10}(2)$ is obviously normal, and the quotient group is isomorphic to the finite orthogonal group $O^{+}\left(10, \mathbb{F}_{2}\right)$ (see 12, Theorem 2.9). We have to explain the meaning of the notation $O^{+}\left(10, \mathbb{F}_{2}\right)$. Up to conjugacy, there are only two types of nondegenerate quadratic forms over $\mathbb{F}_{2}^{10}$. Modulo 2 , the intersection form on $K_{X}^{\perp}$ is equivalent to the quadratic form

$$
x_{1} x_{2}+x_{3} x_{4}+\cdots+x_{9} x_{10} .
$$

In other words, this quadratic form is of even type; the number of its isotropic vectors (including the null vector) is $2^{4}\left(2^{5}+1\right)$. The notation $O^{+}\left(10, \mathbb{F}_{2}\right)$ is meant to distinguish this quadratic form from the form of odd type $x_{1} x_{2}+x_{3} x_{4}+\cdots+$ $x_{7} x_{8}+x_{9} x_{10}+x_{9}^{2}+x_{10}^{2}$, which has only $2^{4}\left(2^{5}-1\right)$ isotropic vectors.

To prove Theorem 3.5, we use the notation from the proof of Theorem 3.2 . Consider the elliptic fibrations $\left|2 f_{1}\right|$ and $\left|2 f_{2}\right|$ defined by the first two isotropic vectors among $f_{1}, \ldots, f_{10}$. From Proposition 3.1, each of these elliptic fibrations comes from a Halphen surface of index 2, and each $\left|f_{i}\right|$ is reduced to a unique element; we denote these curves by $F_{1} \in\left|f_{1}\right|$ and $F_{2} \in\left|f_{2}\right|$. Let $|D|=\left|2 F_{1}+2 F_{2}\right|$.

Lemma 3.7. The linear system $|D|=\left|2 F_{1}+2 F_{2}\right|$ has no base points and defines a morphism $\phi: S \rightarrow \mathbb{P}^{4}$.

Remark 3.8. The proof of this lemma does not use the fact that $-2 K_{X}$ is effective; it depends only on the intersection properties of $f_{1}, f_{2}$ and $K_{X}$, and the fact that $\left|f_{i}\right|=\left\{F_{i}\right\}$ for some irreducible curve. This will be used in Section 5.5 (see Lemma 5.7).

Proof. Consider the following three exact sequences:

$$
\begin{aligned}
& 0 \rightarrow \mathcal{O}_{X}\left(F_{1}\right) \rightarrow \mathcal{O}_{X}\left(F_{1}+F_{2}\right) \rightarrow \mathcal{O}_{F_{1}}\left(F_{1}+F_{2}\right) \rightarrow 0, \\
& 0 \rightarrow \mathcal{O}_{X}\left(F_{1}+F_{2}\right) \rightarrow \mathcal{O}_{X}\left(2 F_{1}+F_{2}\right) \rightarrow \mathcal{O}_{F_{1}}\left(2 F_{1}+F_{2}\right) \rightarrow 0, \\
& 0 \rightarrow \mathcal{O}_{X}\left(F_{1}+2 F_{2}\right) \rightarrow \mathcal{O}_{X}\left(2 F_{1}+2 F_{2}\right) \rightarrow \mathcal{O}_{F_{1}}\left(2 F_{1}+2 F_{2}\right) \rightarrow 0 .
\end{aligned}
$$

Since $\operatorname{deg} \mathcal{O}_{F_{1}}\left(F_{1}+F_{2}\right)=1$ and $p_{a}\left(F_{1}\right)=1$, we get $h^{0}\left(\mathcal{O}_{F_{1}}\left(F_{1}+F_{2}\right)\right)=1$; similarly, $h^{0}\left(\mathcal{O}_{F_{1}}\left(F_{2}\right)\right)=1$.

Since $h^{0}\left(\mathcal{O}_{X}\left(F_{1}\right)\right)=1$, the Riemman-Roch formula implies that $h^{1}\left(\mathcal{O}_{X}\left(F_{1}\right)\right)=$ 0 . Thus the first exact sequence shows that $h^{0}\left(\mathcal{O}_{X}\left(F_{1}+F_{2}\right)\right)=2$, and, by RiemannRoch, $h^{1}\left(\mathcal{O}_{X}\left(F_{1}+F_{2}\right)\right)=0$. Since $\operatorname{deg} \mathcal{O}_{F_{1}}\left(2 F_{1}+F_{2}\right)=1$, the second exact sequence gives $h^{0}\left(\mathcal{O}_{X}\left(2 F_{1}+F_{2}\right)\right)=3$. By Riemann-Roch, $h^{1}\left(\mathcal{O}_{X}\left(2 F_{1}+F_{2}\right)\right)=0$. Since 
$\operatorname{deg} \mathcal{O}_{F_{1}}\left(2 F_{1}+2 F_{2}\right)=2$, the third exact sequence gives $h^{0}\left(\mathcal{O}_{X}\left(2 F_{1}+2 F_{2}\right)\right)=5$; hence $\operatorname{dim}|D|=4$.

Since $F_{1}$ and $F_{2}$ are irreducible and $h^{0}\left(\mathcal{O}_{X}\left(2 F_{1}+2 F_{2}\right)\right)>h^{0}\left(\mathcal{O}_{X}\left(F_{i}+2 F_{j}\right)\right)$, we obtain that $|D|$ has no fixed components. Let us now assume that $|D|$ has a base point. Such a point must lie on $F_{1}$ or $F_{2}$ and, without loss of generality, we may assume that it lies on $F_{1}$. The third exact sequence shows that the restriction map

$$
H^{0}\left(\mathcal{O}_{X}\left(2 F_{1}+2 F_{2}\right)\right) \rightarrow H^{0}\left(\mathcal{O}_{F_{1}}\left(2 F_{1}+2 F_{2}\right)\right)
$$

is a surjective morphism onto a complete linear system of degree 2 on $F_{1}$. Thus $|D|$ has no base point on $F_{1}$, and $|D|$ has no base point at all.

Lemma 3.9. The image of $\phi: X \rightarrow \mathbb{P}^{4}$ is a surface $S$ of degree 4 .

Proof. Since the map is given by the complete linear system $|D|$, its image spans $\mathbb{P}^{4}$ and its degree divides $D^{2}=8$. So, there are only two possibilities: $\phi$ is 1 -to- 1 and its image has degree 8 , and $\phi$ is 2 -to- 1 and its image has degree 4 ; its image cannot have degree $\leq 2$ because every quadric surface of $\mathbb{P}^{4}$ is contained in a hyperplane.

We know that $\left|F_{1}+F_{2}\right|$ is an irreducible pencil. Let $P$ be its general member. It is an irreducible curve of arithmetic genus 2. Let $\omega_{P}$ be its canonical sheaf. Since $\mathcal{O}_{F_{i}}(C) \cong \mathcal{O}_{F_{i}}\left(-2 K_{X}\right) \cong \mathcal{O}_{F_{i}}$, the adjunction formula gives

$$
\omega_{P}^{\otimes 2} \cong \mathcal{O}_{P}\left(2 F_{1}+2 F_{2}+2 K_{X}\right) \cong \mathcal{O}_{P}\left(2 F_{1}+2 F_{2}\right) .
$$

The exact sequence

$$
0 \rightarrow \mathcal{O}_{X}\left(F_{1}+F_{2}\right) \rightarrow \mathcal{O}_{X}\left(2 F_{1}+2 F_{2}\right) \rightarrow \mathcal{O}_{P}\left(2 F_{1}+2 F_{2}\right) \rightarrow 0
$$

shows that $|D|$ cuts out on $P$ the bicanonical linear system; hence the map $\phi_{\mid P}$ is of degree 2 onto a plane conic. In particular, the degree of $\phi: X \rightarrow \phi(X)$ cannot be equal to 1 ; hence it is of degree 2 onto a quartic surface $S$ in $\mathbb{P}^{4}$.

Recall that an anticanonical del Pezzo surface $S$ of degree $d \geq 3$ is a surface of degree $d$ in $\mathbb{P}^{d}$ whose minimal resolution is isomorphic to the blowup $V$ of $9-d$ points in $\mathbb{P}^{2}$ (maybe infinitely near) with $-K_{V}$ nef and big. Each such surface $S$ is obtained as the image of $V$ by the map given by the linear system $\left|-K_{V}\right|$. An anticanonical del Pezzo surface may have singularities (when $-K_{V}$ is not ample). They are Du Val singularities (or ADE-singularities).

It is classically known that a surface of degree 4 in $\mathbb{P}^{4}$ that spans $\mathbb{P}^{4}$ is either an anticanonical del Pezzo surface, or a cone over an elliptic curve, or a projection of a surface of degree 4 in $\mathbb{P}^{5}$ (see [34, 35] and [18). Since $X$ is rational, and $\phi$ is given by a complete linear system there is only one possibility: $S$ is an anticanonical del Pezzo surface.

Lemma 3.10. The image $S$ of $X$ under the map $\phi: X \rightarrow \mathbb{P}^{4}$ is a del Pezzo surface of degree 4 with four ordinary double points.

Proof. Consider the four curves $F_{1}, F_{2}, E_{1}, E_{2}$, where $E_{i}$ is the $(-1)$-curve corresponding to the points $p_{i}, i=1,2$, in the Coble set defining $X$. By definition, $F_{i} \cdot E_{i}=0, i=1,2$, and $F_{i} \cdot E_{j}=1, i \neq j$. Moreover, $2 E_{i}+C \in\left|2 F_{i}\right|$, where $C \in\left|-2 K_{X}\right|, C \cdot F_{i}=0$, and $C \cdot E_{j}=2, i \neq j$.

The restriction of $|D|$ to each of the curves $F_{i}$ is of degree 2 and cuts out a complete linear system of degree 2 on these curves of arithmetic genus 1 ; thus $\phi_{\mid F_{i}}$ is a 2 -to- 1 cover of $F_{i}$ onto a line $\ell_{i}, i=1,2$. 
The restriction of $|D|$ to each of the curves $E_{i}$ is also of degree 2. Since $F_{i} \cdot C=0$ and $C^{2}=-4$, the morphism $\phi$ contracts $C$ onto a singular point $q$ of $S$. This point is contained in $\phi\left(E_{1}\right) \cap \phi\left(E_{2}\right)$ and is a ramification point for the maps $\phi_{\mid E_{i}}: E_{i} \rightarrow$ $\phi\left(E_{i}\right)$. Thus, the images of $E_{1}$ and $E_{2}$ are two lines $\ell_{3}$ and $\ell_{4}$ that intersect at the singular point $q$.

We infer from the exact sequences (14) that

$$
3=h^{0}\left(D-F_{1}\right)>h^{0}\left(D-F_{1}-F_{2}\right)=2 ;
$$

hence $\ell_{1} \neq \ell_{2}$. Similarly, we prove that

- the four lines $\ell_{i}$ are distinct;

- the intersections $\ell_{1} \cap \ell_{2}, \ell_{2} \cap \ell_{3}, \ell_{3} \cap \ell_{4}, \ell_{4} \cap \ell_{1}$ are nonempty;

- all other intersections of two of these lines are empty.

Let $\Pi_{1}$ be the plane spanned by $\ell_{1}$ and $\ell_{2}$ and $\Pi_{2}$ be the plane spanned by $\ell_{3}$ and $\ell_{4}$; the plane $\Pi_{2}$ contains the singularity $q=\ell_{3} \cap \ell_{4}=\phi(C)$. Since $\Pi_{1}$ and $\Pi_{2}$ intersect in at least two points and do not coincide (for $\ell_{1} \cap \ell_{3}=\emptyset$ ) they span a hyperplane $H$ in $\mathbb{P}^{4}$. The pre-image of $H \cap S$ in $X$ is the divisor $F_{1}+F_{2}+C+E_{1}+C+E_{2} \in\left|2 F_{1}+2 F_{2}\right|$.

It is known that a quartic del Pezzo surface is equal to the base locus of a pencil of quadrics. The quadrangle of lines is equal to the base locus of the restriction of the pencil to $H$. It is easy to see that this pencil must be spanned by two quadrics of rank 2, i.e. the union of two planes. It follows from this that the pencil of quadrics containing $S$ is spanned by two quadrics of rank 3 . This implies that $S$ contains 4 singular points of type $A_{1}$ (ordinary nodes) or 2 singular points of type $A_{1}$ and one singular point of type $A_{3}$ (see [12, Lemma 0.4.2). In the second case the surface does not contain a quadrangle of lines. Thus we obtain that $S$ is a 4-nodal quartic del Pezzo surface. Its four nodes are the vertices of the quadrangle of lines. It is known that a 4-nodal quartic del Pezzo surface is isomorphic to the anticanonical model of the blowup of 5 points $p_{1}, \ldots, p_{5}$ such that $p_{3}$ is infinitely near $p_{2}, p_{5}$ is infinitely near $p_{4}$ and the points $p_{1}, p_{2}, p_{3}$ and $p_{1}, p_{4}, p_{5}$ are collinear (see [12, Proposition 0.4.3). The quadrangle of lines is formed by the images of the classes $e_{1}, e_{3}, e_{5}$, and $e_{0}-e_{2}-e_{4}$.

Proof of Theorem 3.5. Let $\sigma: S^{\prime} \rightarrow S$ be the blowup of the point $q=\phi(C)$. The exceptional curve is a (-2)-curve $R$ on $S^{\prime}$. The morphism $\phi$ factors through a finite map $\phi^{\prime}: X \rightarrow S^{\prime}$ of degree 2. The pre-image of $R$ on $X$ is the curve $C$.

Let $\tau$ be the automorphism of $X$ defined by the deck transformation of the finite double cover $\phi^{\prime}: X \rightarrow S^{\prime}$. Note that it is defined even when $\operatorname{char}(\mathbb{K})=2$. Since the genus 1 fibration $X$ is not a quasi-elliptic fibration (the image of its general member is a conic, but an elliptic curve cannot be mapped to a rational curve by an inseparable map), the map $\phi^{\prime}$ is a separable finite morphism.

The Picard group $\operatorname{Pic}\left(S^{\prime}\right) \otimes \mathbb{Q}$ of $\mathbb{Q}$-divisor classes on $S^{\prime}$ is of rank 3. It is generated by the classes of the curve $R$ and the proper transforms of the lines $\ell_{1}$ and $\ell_{2}$. For any divisor class $A$ on $X$, we have, in $\operatorname{Pic}(X) \otimes \mathbb{Q}$,

$$
A+\tau(A)=a_{1} f_{1}+a_{2} f_{2}+a_{3} K_{X} .
$$

Suppose $A \in\left(\mathbb{Z} f_{1}+\mathbb{Z} f_{2}+\mathbb{Z} K_{X}\right)^{\perp}$. Since $f_{1}, f_{2}$ and $K_{X}$ are $\tau$-invariant, we obtain, by intersecting both sides with $f_{1}, f_{2}$, and $K_{X}$, that $a_{1}=a_{2}=a_{3}=0$. Thus $\tau(A)=-A$. The sublattice of $K_{X}^{\perp}$ spanned by $f_{1}$ and $f_{2}$ is isomorphic to the 
hyperbolic plane $\mathbb{H}$. Its orthogonal complement is isomorphic to the lattice $\mathbb{E}_{8}$. Thus we obtain

$$
\tau^{*}=\operatorname{id}_{\mathbb{H}} \oplus-\operatorname{id}_{\mathbb{E}_{8}} .
$$

Now let $w$ be an element of $W_{X}$, and $f_{i}^{\prime}=w\left(f_{i}\right), i=1,2$. Since $X$ has no $(-2)$-curves, we can apply Lemma 3.4 to obtain that the $f_{i}^{\prime}$ are primitive, isotropic and effective divisor classes; since $w$ preserves both $K_{X}$ and the intersection form, $f_{i}^{\prime} \cdot K_{X}=0$ and $f_{1}^{\prime} \cdot f_{2}^{\prime}=1$. Similarly, each class $w\left(\left[E_{i}\right]\right), i=1,2$, is represented by a unique $(-1)$-curve $E_{i}^{\prime}$. The curve $E_{i}^{\prime}$ does not intersect $f_{i}^{\prime}$ and if one contracts $E_{i}^{\prime}$ and applies Proposition [3.1, one sees that $f_{i}^{\prime}$ is the pull-back of a Halphen pencil of index 2. As above we deduce that each linear system $\left|2 f_{i}^{\prime}\right|$ is an elliptic pencil, and repeating the argument by taking $\left(f_{1}^{\prime}, f_{2}^{\prime}\right)$ instead of $\left(f_{1}, f_{2}\right)$, we obtain an automorphism $\tau_{\omega}=w \circ \tau \circ w^{-1}$. This shows that $\operatorname{Aut}(X)^{*}$ contains the minimal normal subgroup containing the involution $\operatorname{id}_{\mathbb{H}} \oplus-\mathrm{id}_{\mathbb{E}_{8}}$. This finishes the proof of Theorem 3.5 because this normal subgroup is isomorphic to $W_{10}(2)$ (this nontrivial result is due to A. Coble; a modern proof can be found in [12, Theorem 2.10.1).

Remark 3.11. One can show that, for a general Coble surface in the moduli sense, the group of automorphisms is isomorphic to $W_{10}(2)$.

Remark 3.12. Assume $\operatorname{char}(\mathbb{K}) \neq 2$. Let $p: X^{\prime} \rightarrow X$ be the double cover of $X$ branched along the curve $C \in\left|-2 K_{X}\right|$. This is a K3-surface which admits an involution whose fixed-point locus consists of a $(-2)$-curve. Conversely, the quotient of a K3-surface by such an involution is a Coble surface. The Picard lattice of $X^{\prime}$ contains the pre-image of the Picard lattice of $X$. It is a primitive sublattice isomorphic to the lattice $\langle-2\rangle \perp \mathbb{E}_{10}(2)$, where $\langle-2\rangle$ is given by the matrix $(-2)$ and $\mathbb{E}_{10}(2)$ is obtained from the lattice $\mathbb{E}_{10}$ by multiplying the values of the quadratic form by 2 .

Over $\mathbb{C}$, it follows from the theory of periods of complex K3 surfaces that the coarse moduli space of such K3 surfaces exists and is an irreducible variety of dimension 9. In fact, it is one of the two codimension 1 irreducible components in the boundary of a compactification of the moduli space of Enriques surfaces. A Coble surface can be obtained as a degeneration of an Enriques surface when its K3cover acquires an ordinary double point. The theory of periods also provides another proof of Theorem 3.5 (see [37, Theorem 10.1.2) and shows that the automorphism group of an unnodal Enriques surface contains a subgroup isomorphic to $W_{10}(2)$ and that a general Enriques surface has its automorphism group isomorphic to $W_{10}(2)$.

Our proof extends to the case of unnodal Enriques surfaces $X$ over a field $\mathbb{K}$ of arbitrary characteristic. In this case any embedding of $\mathbb{H}$ in $\operatorname{Num}(X) \cong \mathbb{H} \perp \mathbb{E}_{8}$ defines a separable map of degree 2 on a 4-nodal quartic surface (it is separable for the same reason as above: the surface has no quasi-elliptic fibrations). The deck transformation acts by formula (16), and we finish as in the case of Coble surfaces by invoking Theorem 2.10.1 from [12].

\section{Gizatullin's Theorem and Cremona special point sets OF NINE POINTS}

Let $X$ denote, as above, a rational surface over $\mathbb{K}$; we denote by $n+1$ its Picard number. In this section, we prove that Cremona special point sets with $n=9$ points are Halphen sets. 
4.1. Parabolic automorphisms. Let $g$ be an automorphism of $X$ and $g^{*}$ the linear transformation of $\operatorname{Pic}(X)$ induced by $g$. Since the intersection form on $\operatorname{Pic}(X)$ has signature $(1, n)$ and the nef cone is $\operatorname{Aut}(X)$-invariant, there are three possibilities for the isometry $g^{*}$ (see [5], 23]).

- $g^{*}$ preserves an ample class $h$, i.e. $g^{*} h=h$; in this case, a positive iterate $g^{k}$ of $g$ acts trivially on $\operatorname{Pic}(X)$ and there is an embedding of $X$ into a projective space $\mathbb{P}_{\mathbb{K}}^{N}$ such that $g^{k}$ extends to a projective linear transformation of $\mathbb{P}_{\mathbb{K}}^{N}$.

- $g^{*}$ preserves a primitive nef class $h$ with $h^{2}=0$ but does not preserve any ample class.

- $g^{*}$ does not preserve any nef class and the spectral radius (i.e. the largest possible absolute value of an eigenvalue) of the linear transformation $g^{*}$ on $\operatorname{Pic}(X) \otimes \mathbb{R}$ is larger than 1 .

In the first case, one says that $g$ (or $g^{*}$ ) is elliptic, in the second that $g$ is parabolic, and in the third that $g$ is hyperbolic (or loxodromic).

When $g$ is parabolic, the class $h$ is, up to a scalar multiple, the unique $g^{*}$-invariant class in the isotropic cone of the intersection form. In particular, the assumption that $h$ is nef could be removed from the definition (see [23], Proposition 4, or [5]). The following fundamental theorem of M. Gizatullin describes geometric properties of parabolic automorphisms.

Theorem 4.1 (M. Gizatullin). Let $X$ be a rational surface over an algebraically closed field $\mathbb{K}$. Let $G \neq\left\{\operatorname{id}_{X}\right\}$ be a group of automorphisms of $X$ such that all elements $g$ in $G \backslash\left\{\operatorname{id}_{X}\right\}$ are parabolic.

Then there exists a unique $G$-invariant primitive nef class $h$ in $\operatorname{Pic}(X)$ with $h^{2}=h \cdot K_{X}=0$ and there exists a unique positive integer $m$ such that the linear system $|m h|$ is an irreducible pencil of curves of arithmetic genus 1 . The relative minimal model of this genus 1 fibration is a Halphen surface $Y$ of index $m$.

Remark 4.2. Assume that $\mathbb{K}=\mathbb{C}$ is the field of complex numbers. Then, Gizatullin's Theorem can be strengthened: a finite index subgroup of $G$ preserves each fiber of the genus one fibration; it acts as a group of translations along the fibers, with dense orbits in almost all fibers; if $G$ is cyclic, periodic orbits are dense in $X$ (see [8, 6]).

Remark 4.3. Gizatullin's Theorem is stated in [23] under the assumption that $\operatorname{char}(\mathbb{K}) \neq 2,3$. As the author points out himself, this assumption is made only to avoid quasi-elliptic fibrations, for which the arguments must be slightly modified but the same conclusion holds. Moreover, in our applications, quasi-elliptic fibrations are not realized.

Corollary 4.4. Let $X$ and $G$ be as in Theorem 4.1. The group $G$ descends to a subgroup of the group $\operatorname{Aut}(Y)$. In particular, it contains a finite index free abelian subgroup $G_{0}$, the rank of which is at most 8 and is equal to 8 if and only if $Y$ is an unnodal Halphen surface.

Proof. This follows from Theorems 4.1 and 2.10.

\subsection{Cremona special implies unnodal.}

Proposition 4.5. A Cremona special rational surface $X$ does not contain (-2)curves; i.e. it is unnodal. 
Proof. Denote by $\mathcal{P}=\left\{p_{1}, \ldots, p_{n}\right\}$ a point set such that $X$ is the blowup of $\mathbb{P}^{2}$ at $\mathcal{P}$; this provides a natural morphism $\pi: X \rightarrow \mathbb{P}^{2}$, and a geometric basis $\left(e_{0}, \ldots, e_{n}\right)$ of $\operatorname{Pic}(X)$. Let $\mathcal{R}_{X}$ be the set of effective real roots (see $₫ 3.3$ ). Our first goal is to prove that $\mathcal{R}_{X}$ is empty.

Let $W_{X}^{\text {nod }}$ be the subgroup of $W_{X}$ generated by the reflections $s_{\alpha}$ with $\alpha \in \mathcal{R}_{X}$. Since all reflections generating $W_{X}^{\text {nod }}$ are conjugate under $W_{X}$ to reflections defined by the simple roots $\alpha_{i}$, a result of Deodhar [14] shows that $W_{X}^{\text {nod }}$ is a Coxeter subgroup of the Coxeter group $W_{X}$. Its intersection with $\operatorname{Aut}(X)^{*}$ is equal to $\{1\}$ ([16], Proposition 35). So, if $\operatorname{Aut}(X)^{*}$ is of finite index in $W_{X}$, the group $W_{X}^{\text {nod }}$ must be finite, and $W_{X}^{\text {nod }}$ is a finite Coxeter group. It follows from the classification of such groups that $\mathcal{R}_{X}$ is a finite set and the sublattice $\mathcal{N}_{X}$ generated by this set is a negative definite sublattice of $K_{X}^{\perp}$; hence $\mathcal{N}_{X} \otimes \mathbb{R}$ is a proper subspace of $K_{X}^{\perp} \otimes \mathbb{R}$.

The group $\operatorname{Aut}(X)^{*}$ leaves the sublattice $\mathcal{N}_{X}$ invariant and acts as a finite group on it. Identifying $K_{X}^{\perp} \otimes \mathbb{R}$ with $\mathbb{E}_{n} \otimes \mathbb{R}, \operatorname{Aut}(X)^{*}$ determines a finite index subgroup of $W_{n}$ that preserves the proper subspace $\mathcal{N}_{X} \otimes \mathbb{R}$.

Assume $n \geq 10$. We invoke a theorem of Yves Benoist and Pierre de la Harpe from 4 according to which the image of $W_{n}$ in $\mathrm{O}\left(\mathbb{E}_{n} \otimes \mathbb{R}\right)$ is Zariski dense. Since, by assumption, $\operatorname{Aut}(X)^{*}$ is a subgroup of finite index in $W_{n}$, we obtain that the image of $\operatorname{Aut}(X)^{*}$ is Zariski dense either in $\mathrm{O}\left(\mathbb{E}_{n} \otimes \mathbb{R}\right)$ or in its connected component of the identity $\operatorname{SO}\left(\mathbb{E}_{n} \otimes \mathbb{R}\right) 6$ Thus the representation of $\operatorname{Aut}\left(X^{*}\right)$ in $\mathbb{E}_{n} \otimes \mathbb{R}$ is irreducible, and hence $\mathcal{N}_{X}=\{0\}$.

When $n=9$, the theorem proved by Benoist and de la Harpe asserts that the image of the Coxeter group $W_{9}$ is Zariski dense in the subgroup $G_{9}$ of $\mathrm{O}\left(\mathbb{E}_{9} \otimes \mathbb{R}\right)$ defined by $G_{9}=\left\{g \in \mathrm{O}\left(\mathbb{E}_{9} \otimes \mathbb{R}\right) \mid g\left(k_{9}\right)=k_{9}\right\}$. If $N$ is a proper subspace of $\mathbb{E}_{9} \otimes \mathbb{R}$ which is fixed by a finite index subgroup of $G_{9}$, then $N / \mathbb{R} k_{9}$ is trivial. As a consequence, $\mathcal{N}_{X}=\{0\}$ in this case too.

Now we may assume that $\mathcal{R}_{X}$ is empty; in particular, no $\alpha_{i}$ is an effective divisor class. Suppose we have a $(-2)$-curve $R$; it represents an effective root which is not a real root. Write $r=[R]=a_{0} e_{0}-\sum a_{i} e_{i} \in \mathcal{R}_{X}$. After permuting the elements of the basis, we may assume that $a_{1} \geq \cdots \geq a_{n}$. Intersecting with $e_{i}$, we obtain that $a_{i} \geq 0$. Applying Noether's inequality [20], Chapter V, Proposition 5, we get $a_{0}<a_{1}+a_{2}+a_{3}$. Since $\alpha_{0}=e_{0}-e_{1}-e_{2}-e_{3}$ is not effective, the three points $p_{1}, p_{2}, p_{3}$ are not collinear. Let $X_{123}$ be the surface obtained by blowing up $\mathbb{P}^{2}$ at $p_{1}, p_{2}$, and $p_{3}$. Let $\sigma$ be the standard quadratic involution with fundamental points $p_{1}, p_{2}, p_{3}$. This birational transformation of the plane lifts to an automorphism of $X_{123}$; thus, the composition of $\pi$ with $\sigma$ is a new birational morphism from $X$ to $\mathbb{P}^{2}$, which provides a new geometric basis for $\operatorname{Pic}(X)$. In terms of the first geometric basis $\left(e_{i}\right)$ of $\operatorname{Pic}(X)$, the change of basis corresponds to the linear transformation $s_{\alpha_{0}}$. As a consequence, if $r$ is the class of a $(-2)$-curve, then the class $r^{\prime}$, defined by

$$
r^{\prime}=s_{\alpha_{0}}(r)=a_{0}^{\prime} e_{0}-\sum_{i=1}^{n} a_{i}^{\prime} e_{i}
$$

\footnotetext{
${ }^{5}$ The assertion is stated for the subgroup generated by the reflections with respect to all $(-2)$ curves, but the proof is extended verbatim to our situation.

${ }^{6} \mathrm{SO}\left(\mathbb{E}_{n} \otimes \mathbb{R}\right)$ is the connected component containing the identity for the Zariski topology, but has two connected components as a real Lie group.
} 
is also the class of a (-2)-curve (in the new geometric basis). Moreover

$$
a_{0}^{\prime}=2 a_{0}-a_{1}-a_{2}-a_{3}, a_{1}^{\prime}=a_{0}-a_{2}-a_{3}, a_{2}^{\prime}=a_{0}-a_{1}-a_{3}, a_{3}^{\prime}=a_{0}-a_{1}-a_{2},
$$

and $a_{i}^{\prime}=a_{i}, i>3$. Now, by Noether's inequality, we have $a_{0}^{\prime}<a_{0}$. Since $s_{\alpha_{0}}$ is in $W_{X}$, we can iterate this process, keeping the assumption that $\mathcal{R}_{X}$ is empty. We can therefore decrease $a_{0}$ until it becomes 0 . In this case one of the simple roots $\alpha_{i}$ becomes effective, a contradiction.

\subsection{Special point sets of nine points.}

Theorem 4.6. Let $\mathcal{P}$ be a Cremona special point set with 9 points. Then $\mathcal{P}$ is an unnodal Halphen set of some index $m$.

Proof. Let $X$ be the surface obtained by blowing up $\mathcal{P}$. Since $\operatorname{Aut}(X)^{*}$ has finite index in $W_{X}$, this group contains also a free abelian group of rank 8 equal to a subgroup of finite index in $\iota\left(\mathbb{E}_{8}\right)$ (see 92.4$)$. This group preserves the isotropic vector $K_{X}$. By Gizatullin's theorem there exists a (minimal) positive integer $m$ such that the linear system $\left|-m K_{X}\right|$ is an irreducible elliptic pencil. Thus $X$ is a Halphen surface of index $m$. By Proposition 4.5, $X$ has no $(-2)$-curves. Thus $X$ is an unnodal Halphen surface.

\section{THE GENERAL CASE}

The main results of this section and the next one are summarized in the following two theorems.

Theorem 5.1. Let $\mathcal{P}$ be a point set of $n \geq 10$ points which is not contained in a cubic curve. If $\mathcal{P}$ is Cremona special, then $\mathcal{P}$ is an unnodal Coble set. In particular, $n$ is equal to 10 .

By definition, a Harbourne set is a set $\mathcal{P}$ of $n \geq 9$ nonsingular points on a cuspidal cubic $C_{0}$ over a field of positive characteristic with the following property: If $X$ denotes the surface obtained by blowing up $\mathcal{P}$ and $C$ denotes the strict transform of $C_{0}$ in $X$, then $X$ has no $(-2)$-curves and the restriction morphism $\mathfrak{r}$ from $K_{X}^{\frac{1}{X}}$ to $\operatorname{Pic}^{0}(C)$ has finite image. These point sets are Cremona special; this is proved by Harbourne in [24].

Theorem 5.2. Let $\mathcal{P}$ be a point set of $n \geq 10$ points contained in a cubic curve $C_{0} \subset \mathbb{P}^{2}$. If $\mathcal{P}$ is Cremona special, then $\mathbb{K}$ has positive characteristic, $C_{0}$ is a cuspidal cubic, and $\mathcal{P}$ is a Harbourne set.

These results imply the Main Theorem. In this section, we prove Theorem 5.1

5.1. A surface $Y$ with ten Halphen pencils. Let $\mathcal{P}=\left\{p_{1}, \ldots, p_{n}\right\}$ be a Cremona special set, with the convention that $j>i$ if $p_{j}$ is infinitely near $p_{i}$. We blow up successively each point $p_{i}$ and denote by

$$
X=X_{n} \rightarrow X_{n-1} \rightarrow \cdots \rightarrow X_{1} \rightarrow \mathbb{P}^{2}
$$

the sequence of blowups. Let $q_{i}: X \rightarrow X_{i}$ be the corresponding birational morphism and $q_{i}^{*}: \operatorname{Pic}\left(X_{i}\right) \rightarrow \operatorname{Pic}(X)$ be the canonical homomorphism of the Picard groups. Obviously $q_{i}^{*}\left(K_{X_{i}}^{\perp}\right) \subset K_{X}^{\perp}$. Moreover the image under $q_{i}^{*}$ of the geometric basis of $\operatorname{Pic}\left(X_{i}\right)$ (defined by the morphism $X_{i} \rightarrow \mathbb{P}^{2}$ ) coincides with the first $i+1$ vectors of the geometric basis of $X$ (defined by the morphism $X \rightarrow \mathbb{P}^{2}$ ). This allows one to identify $W_{X_{i}}$ with a subgroup of $W_{X}$. Since $\operatorname{Aut}(X)^{*}$ is of finite index in $W_{X}$, 
its intersection with $W_{X_{i}}$ is a subgroup of finite index; this subgroup preserves the exceptional curves obtained from the blowups of $p_{j}$ for $j>i$, and descends as a group of automorphisms of $X_{i}$. Thus all surfaces $X_{i}, i \geq 9$, are Cremona special, and all sets $\left\{p_{1}, \ldots, p_{i}\right\}, i \geq 9$, are Cremona special sets. By Theorem 4.6, the surface $X_{9}$ is an unnodal Halphen surface. In particular, the first 9 points do not contain infinitely near points. Since the set of the first 10 points is Cremona special, Proposition 4.5 shows that the surface $X_{10}$ has no $(-2)$-curves; hence $p_{10}$ is not infinitely near $p_{i}$ for $i \leq 9$ and the set $\left\{p_{1}, \ldots, p_{10}\right\}$ contains no infinitely near point.

Let $i$ be an index between 1 and 10 such that $p_{11}$ is not infinitely near $p_{i}$. Consider the sequence of points $p_{1}, \ldots, p_{i-1}, p_{i+1}, \ldots, p_{11}$, and apply the same argument. We obtain that $p_{11}$ is not infinitely near the points $p_{j}$ for $1 \leq j \leq 10$. By induction on $n$, and permutation of the points, this proves the following lemma.

Lemma 5.3. If $\mathcal{P}$ is a Cremona special point set of $n \geq 9$ points, then $\mathcal{P}$ is a proper subset of $n$ distinct points in $\mathbb{P}^{2}$, and all subsets of $\mathcal{P}$ of $m \geq 9$ points are Cremona special.

Let $Y=X_{10}, E_{i} \subset Y$ be the (-1)-curve obtained by blowing up $p_{i}, 1 \leq i \leq 10$, and $\sigma_{E_{i}}: Y \rightarrow Y_{i}$ be the blowdown of $E_{i}$. Since $Y_{i}$ is Cremona special, it is an unnodal Halphen surface of some index $m_{i}$. The pre-image of the elliptic fibration of $Y_{i}$ is an elliptic pencil $\left|m_{i} f_{i}\right|$ on $Y$, where

$$
f_{i}=-K_{Y}+e_{i}=\sigma_{E_{i}}^{*}\left(-K_{Y_{i}}\right) .
$$

The divisor classes $f_{1}, \ldots, f_{10}$ are exactly the primitive isotropic vectors in $K_{Y}^{\perp}$ which we introduced in the proof of Theorem 3.2 . We denote by $\tilde{p}_{i} \in Y_{i}$ the point $\sigma_{E_{i}}\left(E_{i}\right)$; this point is the pre-image of $p_{i}$ under the natural projection $Y_{i} \rightarrow \mathbb{P}^{2}$.

5.2. Almost all indices are different from 1. Suppose that two indices $m_{i}$ and $m_{j}$ are equal to 1 , say $m_{1}=m_{2}=1$. Let $B_{i} \subset Y_{i}, i=1,2$, be the fiber of the Halphen fibration which contains $\tilde{p}_{i}$, and let $a_{i}$ be the multiplicity of this fiber at $\tilde{p}_{i}$. Let $A_{i} \subset Y$ be the strict transform of $B_{i}$ under the morphism $\sigma_{E_{i}}$. Since the fibers of the Halphen surface $Y_{i}$ are irreducible, both $A_{i}$ and $B_{i}$ are irrreducible. Then $-K_{Y}$ is effective and represented by $A_{i}+\left(a_{i}-1\right) E_{i}$. Since $K_{Y}^{2}=-1$, the two curves $A_{1}+\left(a_{1}-1\right) E_{1}$ and $A_{2}+\left(a_{2}-1\right) E_{2}$ have a common component, and thus $A_{1}=A_{2}$; as a consequence, $a_{1}=a_{2}=1$, i.e. $\tilde{p}_{i}$ is a smooth point of $B_{i}$, for $i=1,2$. Denote by $A$ the curve $A_{1}=A_{2}$. By Lemma 2.4, the normal bundle of $A_{i}$ is equal to $\mathcal{O}_{A_{i}}\left(-E_{i} \cap A_{i}\right), i=1,2$. Thus the points $E_{1} \cap A$ and $E_{2} \cap A$ are linearly equivalent on $A$. Since $p_{1} \neq p_{2}$ and $A$ is an irreducible curve of arithmetic genus 1 , we get a contradiction. This proves the following lemma.

Lemma 5.4. At least nine of the indices $m_{i}, 1 \leq i \leq 10$, are larger than 1 .

5.3. An alternative. Effective curves with divisor class $f_{i}$ are total transforms of fibers of the Halphen fibration on $Y_{i}$ with multiplicity $m_{i}$. Thus, if $m_{i}=1$, the class $f_{i}$ is represented by an irreducible curve (the total transform of any fiber that does not contain $p_{i}$ ), and if $m_{i} \geq 2$ the class $f_{i}$ is represented by an irreducible curve if and only if $\tilde{p}_{i}$ is not contained in the multiple fiber of $Y_{i}$.

Lemma 5.5. Either the set $\left\{p_{1}, \ldots, p_{10}\right\}$ is contained in (the smooth part of) a cubic curve $C$ and then all $f_{i}, 1 \leq i \leq 10$, are represented by $C^{\prime}+E_{i}$, where $C^{\prime}$ is the strict transform of $C$, or $\left\{p_{1}, \ldots, p_{10}\right\}$ is not contained in a cubic curve, and then all classes $f_{i}$ are represented by at least one irreducible curve. 
Proof. If the set $\mathcal{P}_{10}=\left\{p_{1}, \ldots, p_{10}\right\}$ is contained in a cubic curve $C$, no $p_{i}$ is a singular point of $C$, because otherwise the Halphen surface $Y_{j}, j \neq i$, would not be special. If the set $\mathcal{P}_{10}$ is not contained in a cubic curve, then $p_{i}$ is not contained in the multiple fiber of $Y_{i}$, so that $f_{i}$ is represented by an irreducible curve.

For the remainder of this section, we make the following assumption, where Lemma 5.5 is used to prove the equivalence.

(A) Each primitive isotropic class $f_{i}$ is represented by an irreducible curve. Equivalently, there is no irreducible cubic curve containing the set $\left\{p_{1}, \ldots\right.$, $\left.p_{10}\right\}$. In particular, the multiple fiber of $Y_{i}$ does not contain $\tilde{p}_{i}$.

In other words, we now assume that $Y$ satisfies the assumption of Theorem 5.1 . Note that the irreducibility of the cubic curves in Assumption (A) follows from Proposition 4.5 .

5.4. All indices are different from 1. Suppose now that one index, say $m_{1}$, is equal to 1 ; by Lemma 5.4, $m_{i} \geq 2$ for $i \geq 2$. As above, let $B_{1} \subset Y_{1}$ be the fiber of the Halphen fibration which contains the point $\tilde{p_{1}}, a_{1} \leq 2$ be its multiplicity at $\tilde{p_{1}}$, and $A_{1}$ be its strict transform in $Y$; then

$$
\sigma_{E_{1}}^{*}\left(B_{1}\right)=f_{1}=A_{1}+a_{1} E_{1} .
$$

Let $B_{2} \subset Y_{2}$ be the fiber of the Halphen fibration which contains the point $\tilde{p_{2}}$. By our assumption (A), the curve $B_{2}$ is not the multiple fiber of the Halphen surface $Y_{2}$. Let $A_{2}$ be the strict transform of $B_{2}$ and $a_{2}$ be the multiplicity of $B_{2}$ at $\tilde{p}_{2}$. Then

$$
A_{2} \sim m_{2} f_{2}-a_{2} E_{2} \sim m_{2}\left(-K_{Y}+E_{2}\right)-a_{2} E_{2} \sim-m_{2} K_{Y}+\left(m_{2}-a_{2}\right) E_{2}
$$

and

$$
\begin{aligned}
A_{1} \cdot A_{2} & =\left(-K_{Y}+\left(1-a_{1}\right) E_{1}\right) \cdot\left(-m_{2} K_{Y}+\left(m_{2}-a_{2}\right) E_{2}\right) \\
& =-m_{2}+\left(m_{2}-a_{2}\right)+m_{2}\left(1-a_{1}\right) \\
& =-a_{2}-\left(a_{1}-1\right) m_{2} ;
\end{aligned}
$$

this number is negative because $a_{i} \geq 1$. Since $A_{1}$ and $A_{2}$ are irreducible curves, we obtain $A=A_{1}=A_{2}$. Since $A_{1}$ is the strict transform of a cubic curve, this contradicts our standing assumption (A) and proves the following lemma.

Lemma 5.6. Under assumption (A), all indices $m_{i}$ are larger than 1.

5.5. The linear system $|D|=\left|2 f_{1}+2 f_{2}\right|$. We now show that all indices $m_{i}$ are indeed equal to 2 ; permuting the indices, it is sufficient to consider $m_{1}$ and $m_{2}$ : We already know that $m_{1}, m_{2}>1$ and we want to show that $m_{1}=m_{2}=2$. For this purpose, we study the linear system $\left|2 f_{1}+2 f_{2}\right|$.

Since $m_{i} \geq 2$ for all $i$, the linear system $\left|f_{i}\right|$ consists of a unique element. Hence,

$$
\left|f_{1}\right|=\left\{F_{1}\right\}, \quad\left|f_{2}\right|=\left\{F_{2}\right\},
$$

where the divisors $F_{1}$ and $F_{2}$ are irreducible curves of arithmetic genus 1 by assumption (A). Since $F_{1} \cdot F_{2}=1$, the curves $F_{1}$ and $F_{2}$ intersect transversally at one point

$$
y_{0}=F_{1} \cap F_{2} .
$$

Denote by $|D|$ the linear system $\left|2 F_{1}+2 F_{2}\right|$. 
Lemma 5.7. Under assumption (A), the following properties are satisfied.

(i) The linear system $|D|=\left|2 F_{1}+2 F_{2}\right|$ satisfies

$$
D^{2}=8, \operatorname{dim}|D|=4,
$$

it has no fixed component, and it has no base point. It defines a morphism $\phi: Y \rightarrow \mathbb{P}^{4}$.

(ii) Let $S=\phi(Y) \subset \mathbb{P}^{4}$ be its image. Then, on each curve $F_{i}, i=1,2, \phi$ restricts to a 2-to-1 cover onto a line $\ell_{i} \subset S$. The two lines $\ell_{1}$ and $\ell_{2}$ are different.

(iii) The linear system $\left|F_{1}+F_{2}\right|$ is an irreducible pencil with two base points $y_{1} \in F_{1}, y_{2} \in F_{2}$. The points $s_{i}=\phi\left(y_{i}\right), i=0,1,2$, span a plane in the projective space $\mathbb{P}^{4}$.

(iv) The degree of $\phi: Y \rightarrow \mathbb{P}^{4}$ is equal to 2 , and its image is an anticanonical del Pezzo surface $S \subset \mathbb{P}^{4}$ of degree 4 ; in particular, $S$ is a normal surface.

(v) The pre-image of $s_{0}$ under the map $\phi$ consists of exactly one point: $\phi^{-1}\left\{s_{0}\right\}$ $=\left\{y_{0}\right\}$.

Proof. (i) The argument is the same as in the proof of Lemma 3.7 where we used only that $f_{1}$ and $f_{2}$ were represented by irreducible curves $F_{1}$ and $F_{2}$ that do not move (see Remark 3.8).

(ii) Each curve $F_{i}, i=1,2$, is an irreducible curve of arithmetic genus 1 . It follows from the third exact sequence in (14) that the restriction of $|D|$ to the curve $F_{i}$ is given by a complete linear system of degree 2 . Thus, for $i=1,2$, the curve $\phi\left(F_{i}\right)$ is a line $\ell_{i} \subset S$ and $\phi$ restricts to a 2 -to- 1 cover between $F_{i}$ and $\ell_{i}$. The same exact sequences show that $\ell_{1} \neq \ell_{2}$.

(iii) The first exact sequence in (14) shows that $\left|F_{1}+F_{2}\right|$ is a pencil of curves of arithmetic genus 2 . Since $\left(F_{1}+F_{2}\right) \cdot F_{1}=1$, its restriction to $F_{1}$ (resp. to $F_{2}$ ) has degree 1 .

Let $\epsilon_{i}$ be the nontrivial $m_{i}$-torsion divisor class on $F_{i}$ such that $\mathcal{O}_{F_{i}}\left(\epsilon_{i}\right) \cong$ $\mathcal{O}_{F_{i}}\left(F_{i}\right)$. For $i=1,2$ we have $\mathcal{O}_{F_{i}}\left(F_{1}+F_{2}\right) \cong \mathcal{O}_{F_{i}}\left(y_{i}\right)$, where $y_{i}$ is an element of the linear system $\left|y_{0}+\epsilon_{i}\right|$ on $F_{i}$. Clearly, $y_{i} \neq y_{0}$ and does not move because the arithmetic genus of $F_{i}$ is 1 . This shows that $\left|F_{1}+F_{2}\right|$ has no fixed components and its base points are $y_{1}$ and $y_{2}$.

The restriction of $\phi$ to $F_{i}$ is given by the linear system $|D|$, i.e. by

$$
\left|\mathcal{O}_{F_{i}}\left(2 F_{1}+2 F_{2}\right)\right|=\left|\mathcal{O}_{F_{i}}\left(2 y_{0}+2 \epsilon_{i}\right)\right| \text {. }
$$

Since $2 y_{0}+2 \epsilon_{i} \sim y_{i}+y_{0}+\epsilon_{i} \nsim y_{0}+y_{i}$, we obtain that the points $y_{0}$ and $y_{i}$ are not mapped to the same point by $\phi$; thus, $s_{0}=\phi\left(y_{0}\right)$ and $s_{i}=\phi\left(y_{i}\right), i=1,2$, are distinct. As a consequence, the plane $\Pi_{0}$ spanned by the two lines $\ell_{1}$ and $\ell_{2}$ is also spanned by the three points $s_{0}, s_{1}$, and $s_{2}$.

(iv) Let us now prove that the degree of $\phi$ is equal to 2 , i.e. $\operatorname{deg} S=4$. Then, as in Section 3.4 (see after Lemma 3.9), the image $S$ of $\phi$ is a del Pezzo surface of degree 4.

Since the map is given by a complete linear system, and a quadric surface in $\mathbb{P}^{4}$ is contained in a hyperplane, the only other possibility is that the degree is equal to 1, i.e. $\operatorname{deg} S=8$; thus, we now assume $\operatorname{deg}(S)=8$ and seek a contradiction.

Let $\mathfrak{o}$ be a general point of $Y$; in particular, $\mathfrak{o}$ is not a base point of $\left|F_{1}+F_{2}\right|$ and its image $\mathfrak{o}^{\prime}=\phi(\mathfrak{o})$ in $S$ is not contained in the plane $\Pi_{0}$. Let $\pi: S \rightarrow S^{\prime} \subset \mathbb{P}^{3}$ be the projection from $\mathfrak{o}^{\prime}$. The composition $\pi \circ \phi$ is a rational map given by the linear 
subsystem $L$ of $\left|2 F_{1}+2 F_{2}\right|$ of divisors passing through the point $\mathfrak{o}$. Replacing $Y$ by its blowup $Y^{\prime} \rightarrow Y$ at the point $\mathfrak{o}$, we obtain a birational morphism $\phi^{\prime}: Y^{\prime} \rightarrow S^{\prime}$ onto a surface of degree 7 .

A general member $P$ of the pencil $\left|F_{1}+F_{2}\right|$ is an irreducible curve of arithmetic genus 2. Consider the restriction of the map $\phi^{\prime}$ to $P$. It is given by a linear series of degree 4 . Would this linear series coincide with $\left|2 K_{P}\right|$, then $\phi_{\mid P}^{\prime}: P \rightarrow \phi^{\prime}(P)$ would be a 2 -to- 1 cover onto a plane conic (see the proof of Lemma 3.9 ), and $\phi$ would have degree 2. Thus, $\phi_{\mid P}^{\prime}$ is given by a linear series $\left|K_{P}+p+q\right|$, where $p+q \notin\left|K_{P}\right|$. The image $\phi^{\prime}(P)$ is a plane quartic curve, the points $p, q$ are mapped to a singular point of $\phi^{\prime}(P)$, and $\phi_{\mid P}^{\prime}$ provides an isomorphism from $P \backslash\{p, q\}$ to $\phi^{\prime}(P) \backslash\left\{\phi^{\prime}(p)\right\}$.

The unique member $P_{0}$ from $\left|F_{1}+F_{2}\right|$ which passes through the point $\mathfrak{o}$ is mapped to a plane curve on $S$ passing through $\mathfrak{o}^{\prime}$. This curve has degree 4 ; hence it is projected to a triple line $\ell_{0}^{\prime}$ on $S^{\prime}$. Since $y_{1}, y_{2} \in P_{0}$, the triple line $\ell_{0}^{\prime}$ contains the projections $s_{1}^{\prime}, s_{2}^{\prime}$ of the points $s_{1}, s_{2}$. By assertion (iii), the points $s_{0}, s_{1}, s_{2}$ span a plane $\Pi_{0}$ in $\mathbb{P}^{4}$. Since $\Pi_{0}$ does not contain the image $\mathfrak{o}^{\prime}$ it is projected onto a plane $\Pi_{0}^{\prime} \subset \mathbb{P}^{3}$ and this plane is spanned by the images $s_{0}^{\prime}, s_{1}^{\prime}$, and $s_{2}^{\prime}$ of the three points $s_{0}, s_{1}$, and $s_{2}$. The triple line $\ell_{0}^{\prime}=\phi^{\prime}\left(P_{0}\right)$ is spanned by $s_{1}^{\prime}$ and $s_{2}^{\prime}$.

The image of the pencil $\left|F_{1}+F_{2}\right|$ on $S^{\prime}$ is cut out by the pencil $\mathcal{Q}$ of planes containing $\ell_{0}^{\prime}$. The plane $\Pi_{0}^{\prime}$ is a member of $\mathcal{Q}$ and cuts out in $S^{\prime}$ a curve of degree 7: This curve is equal to the union of the three lines $\ell_{1}^{\prime}, \ell_{2}^{\prime}$, and $\ell_{0}^{\prime}$, where the first two enter with multiplicity 2 and the last one with multiplicity 3 . Any other plane from $\mathcal{Q}$ cuts out in $S^{\prime}$ the line $\ell_{0}^{\prime}$ taken with multiplicity 3 and a quartic curve $P^{\prime}=\phi^{\prime}(P)$ for some $P \in\left|F_{1}+F_{2}\right|$. Let $N$ be the closure of the set of double points of irreducible plane quartics cut out by $\mathcal{Q}$ in $S^{\prime}$. It is a double conic $K$ on $S^{\prime}$ (its pre-image on $Y$ intersects $P$ at two points, hence intersects $2 F_{1}+2 F_{2}$ with multiplicity 4$)$. It passes through a point on $\ell_{0}^{\prime}$, the image of a double point of the plane quartic $\phi\left(P_{0}\right)$.

Let us see what else is in the singular locus of $S^{\prime}$. Suppose $Z$ is another irreducible curve in the singular locus of $S^{\prime}$ which is not contained in any plane from $\mathcal{Q}$. Then a general plane $\Pi^{\prime}$ from $\mathcal{Q}$ intersects it, and hence the image of a general $P \in\left|F_{1}+F_{2}\right|$ in $\Pi^{\prime}$ acquires an additional double point. This contradiction shows that $Z$ is contained in some plane $\Pi^{\prime} \in \mathcal{Q}$, certainly, different from $\Pi_{0}^{\prime}$. We claim that it must be a double conic. The unique alternative possibility is that $Z$ is a double line. Let $F$ be its pre-image in $Y$. It is an irreducible component of some divisor $P$ from $\left|F_{1}+F_{2}\right|$, with $P=F+R$ for some effective divisor $R$. Since the line $Z$ intersects only one of the points $s_{1}^{\prime}$ and $s_{2}^{\prime}$, the curve $F$ passes through only one base point of $\left|F_{1}+F_{2}\right|$; hence we may assume that $F \cdot F_{1}=0$. Let $\sigma_{E_{1}}: Y \rightarrow Y_{1}$ be, as in Section 5.1, the blowdown of the curve $E_{1}$. We know that $Y_{1}$ is a Halphen surface with elliptic fibration $\left|m_{1} \sigma_{E_{1}}\left(F_{1}\right)\right|$, the fibers of which are irreducible. We also know that $F_{1}=\sigma_{E_{1}}^{*}\left(-K_{Y_{1}}\right)$; hence $\left(\sigma_{E_{1}}\right)_{*}(F) \cdot K_{Y_{1}}=0$. If $\sigma_{E_{1}}(F)$ is not a point, it must be a fiber of the elliptic fibration $\left|m_{1} \sigma_{E_{1}}\left(F_{1}\right)\right|$, and it cannot be the multiple fiber $\sigma_{E_{1}}\left(F_{1}\right)$ (because $Z \neq \ell_{1}^{\prime}$ ). Since $F \cdot F_{2} \leq 1$, this contradicts $m_{1}>1$, and shows that $\sigma_{E_{1}}(F)$ is a point, which means that $F$ is the curve $E_{1}$. In particular, $F \cdot F_{2}=1, F_{1} \cdot R=1$, and $F_{2} \cdot R=0$, because the self-intersection of $P$ is 2 . The same reasoning, applied to $\sigma_{E_{2}}$, implies that $R$ is equal to $E_{2}$, and provides a contradiction because $E_{1}+E_{2}$ is not a member of $\left|F_{1}+F_{2}\right|$. 
So, we have now computed the singular locus of $S^{\prime}$. It consists of two double lines $\ell_{1}^{\prime}, \ell_{2}^{\prime}$, one triple line $\ell_{0}^{\prime}$, the double conic $K$, and some number $c$ of double conics contained in planes from the pencil $\mathcal{Q}$.

A general plane section of $S^{\prime}$ is a plane curve of degree 7 . It has $2+2(c+1)$ double points and one triple point. Its geometric genus is equal to $15-3-2-2(c+1)$, which is an even number. On the other hand, the geometric genus is equal to the genus of a general curve from $\left|2 F_{1}+2 F_{2}\right|$, which is equal to 5 . This contradiction proves assertion (iv).

(v) Since $\phi: Y \rightarrow S$ is a map of degree 2 onto a normal surface, the existence of the Stein decomposition of $\phi$ implies that the pre-image of any point consists of at most two points or contains a one-dimensional component.

We want to show that the pre-image of $\left\{s_{0}\right\}$ is reduced to $\left\{y_{0}\right\}$. It contains the union of the pre-images of $s_{0}$ under the restriction maps $F_{i} \rightarrow \ell_{i}$. Since each such map is of degree 2 and $\phi$ does not blow down any curve intersecting $F_{1}+F_{2}$, we may assume that $y_{0}$ is a ramification point of $\phi_{\mid F_{1}}: F_{1} \rightarrow \ell_{1}$ and the pre-image of $F_{2} \rightarrow \ell_{2}$ consists of two points $y_{0}$ and $y_{0}^{\prime}$.

Let $\nu: \bar{S} \rightarrow S$ be the blowup of the point $s_{0}$. Suppose $s_{0}$ is a nonsingular point of $S$. Then the exceptional curve $E$ of $\nu$ is a $(-1)$-curve on $\bar{S}$. The rational map $\nu^{-1} \circ \phi: S \rightarrow \bar{S}$ extends to a regular map $\bar{\phi}: \bar{Y} \rightarrow \bar{S}$ with disconnected exceptional locus over $y_{0}$ and $y_{0}^{\prime}$. However, the restriction of $\bar{\phi}$ over $E$ is ramified at the point of intersection of $E$ with the proper transform of $\ell_{1}$, and hence $\bar{\phi}^{-1}(E)$ cannot be disconnected. This contradiction shows that $s_{0}$ is a singular point of $S$; hence $\phi$ is not étale at $y_{0}$, and hence $s_{0}$ is a ramification point of $\phi$ and, as such, has a unique pre-image.

Now we can easily deduce from this lemma that $m_{1}=m_{2}=2$. We know that the maps $F_{i} \rightarrow \ell_{i}$ ramify over $s_{0}$. Each map is given by the restriction of the linear system $\left|2 F_{1}+2 F_{2}\right|$. It is equal to the linear system $\left|2 y_{0}+2 \epsilon_{i}\right|$, where $\mathcal{O}_{F_{i}}\left(\epsilon_{i}\right) \cong \mathcal{O}_{F_{i}}\left(F_{i}\right)$ and $m_{i}$ is the order of $\epsilon_{i}$ in $\operatorname{Pic}^{0}\left(F_{i}\right)$. Since $2 y_{0} \in\left|2 y_{0}+2 \epsilon_{i}\right|$, we obtain $2 \epsilon_{i} \sim 0$; hence $m_{i} \leq 2$. Since all indices $m_{i}$ are larger than one, we get $m_{i}=2$ for all $1 \leq i \leq 10$.

5.6. The surface $Y$ is a Coble surface. The surface $Y$ is obtained from $Y_{i}$ by blowing up a point $\tilde{p}_{i}$; this point is contained in a nonmultiple member $B_{i}$ of the Halphen pencil of $Y_{i}$. Let $a_{i}$ be the multiplicity of $\tilde{p}_{i}$ on $B_{i}$ : it is equal to 1 if $\tilde{p}_{i}$ is a nonsingular point of $B_{i}$ and 2 otherwise. Let $A_{i}$ be the strict transform of $B_{i}$ on $Y$. Since $m_{i}=2$, we have

$$
A_{i}+a_{i} E_{i} \sim 2 f_{i}=-2 \sigma_{E_{i}}^{*}\left(K_{Y_{i}}\right)=-2 K_{Y}+2 E_{i} .
$$

If $a_{i}=2$ for some index, then $A_{i} \in\left|-2 K_{Y}\right|$; hence $Y$ is a Coble surface, all $A_{i}$ coincide, and their natural projection on $\mathbb{P}^{2}$ is a sextic curve with double points at $p_{1}, p_{2}, \ldots, p_{10}$.

Suppose $a_{i}=1$ for all $i$. Then

$$
A_{i} \sim-2 K_{Y}+E_{i} .
$$

Let $F_{i} \in\left|f_{i}\right|=\left|-K_{Y}+E_{i}\right|$ be the pre-image of the reduced double fiber of the Halphen pencil on $Y_{i}$. Since $\sigma_{E_{i}}$ is an isomorphism over an open neighborhood of this fiber, the normal bundle $\mathcal{O}_{F_{i}}\left(F_{i}\right)$ is of order 2 in $\operatorname{Pic}\left(F_{i}\right)$. Applying (18), we obtain

$$
\mathcal{O}_{F_{i}}\left(A_{j}-E_{j}\right) \cong \mathcal{O}_{F_{i}}\left(-2 K_{Y}\right) \cong \mathcal{O}_{F_{i}}
$$


For $j \neq i, A_{j} \cdot F_{i}=E_{j} \cdot F_{i}=1$; hence $A_{j}$ and $E_{j}$ intersect $F_{i}$ transversally at the same point. Let $\pi: Y \rightarrow \mathbb{P}^{2}$ be the natural projection, i.e. the blowdown of all curves $E_{l}, 1 \leq l \leq 10$. The image of $F_{i}$ is an irreducible cubic curve $C_{i}$. By blowing down $E_{j}$, we see that $\pi\left(A_{j}\right)$ is tangent to $C_{i}$ at $p_{j}$. Taking $k \neq i, j$, we obtain that $\pi\left(A_{j}\right)$ is tangent at the same point to $C_{k}$; since $A_{j}$ intersects $E_{j}$ transversely, $C_{k}$ and $C_{i}$ are tangent at $p_{j}$. Fixing $i$ and $k$ and changing $j$, we see that $C_{k}$ and $C_{i}$ are tangent at the 8 points $p_{j}$ with $j \neq k$, so that the two irreducible cubics $C_{i}$ and $C_{k}$ coincide. Hence $F_{i}=F_{k}$, while $f_{i} \neq f_{k}$. This contradiction shows that $Y$ is a Coble surface.

Thus we have proved Theorem 5.1 when $\mathcal{P}$ contains exactly 10 points.

5.7. The surface $X$ coincides with the Coble surface $Y$. To conclude the proof of Theorem 5.1, it remains to show that $n=10$, and thus $X=Y$ is a Coble surface, when $\mathcal{P}$ is a Cremona special set not contained in a cubic curve. We may assume that $X=X_{11}$, so that $X$ is obtained by blowing up 11 points $p_{1}, \ldots, p_{11}$ in $\mathbb{P}^{2}$; by Lemma 5.3 none of them is an infinitely near point.

By assumption, $\mathcal{P}$ is not contained in a cubic curve. Let $\mathcal{P}_{j}, 1 \leq j \leq 11$, be the subset of $\mathcal{P}$ obtained by removing the point $p_{j}$. Assume that three of the sets $\mathcal{P}_{j}$, say $\mathcal{P}_{9}, \mathcal{P}_{10}$ and $\mathcal{P}_{11}$, are contained in cubic curves, say $C_{9}, C_{10}$ and $C_{11}$. By Section 5.3, these three cubics are irreducible. Let $\mathcal{P}^{\prime}=\left\{p_{1}, \ldots, p_{8}\right\}$. Since $\mathcal{P}^{\prime}$ lies on an irreducible cubic curve, no four of its points are collinear. It follows that the linear system of cubic curves containing $\mathcal{P}^{\prime}$ is of dimension 1 ; let $q$ be the ninth base point of this pencil. The curves $C_{9}, C_{10}$, and $C_{11}$ belong to this pencil; hence any two of them intersect at $q$. Consequently $C_{9}$ and $C_{10}$ contain the set $\mathcal{P}^{\prime}$ and the points $q$ and $p_{11}$. It follows that $C_{9}=C_{10}$ or that $q=p_{11}$. If $C_{9}=C_{10}$, then $\mathcal{P}$ is contained in this cubic curve, if $q=p_{11}$, then $C_{11}$ contains $\mathcal{P}$; in both cases, $\mathcal{P}$ is contained in a cubic curve, a contradiction.

Thus, at most 2 of the $\mathcal{P}_{j}$ are contained in cubic curves. We can therefore suppose that $\mathcal{P}_{1}, \mathcal{P}_{2}, \mathcal{P}_{3}$ and $\mathcal{P}_{4}$ are not contained in cubic curves. The surfaces obtained by blowing up these sets are Cremona special and, as such, are unnodal Coble surfaces. In particular, each set $\mathcal{P}_{l}, 1 \leq l \leq 4$, determines a unique curve of degree six with nodes along $\mathcal{P}_{l}$.

Let us first assume that the characteristic of the field $\mathbb{K}$ differs from 2. Consider the del Pezzo surface $Z$ of degree 2 obtained by blowing up the last seven points $p_{5}, \ldots, p_{11}$. We identify the elements of the set

$$
\mathcal{Q}=\left\{p_{1}, p_{2}, p_{3}, p_{4}\right\}
$$

with points on $Z$. By assumption, we have 4 curves $C_{i}$ on $Z$ in the linear system $\left|-2 K_{Z}\right|$ with double points at $\mathcal{Q} \backslash\left\{p_{i}\right\}$; each $C_{i}$ is the proper transform of the sextic curve with nodes along $\mathcal{P}_{i}, 1 \leq i \leq 4$. Consider the map $Z \rightarrow \mathbb{P}^{2}$ defined by the linear system $\left|-K_{Z}\right|$. Since $\operatorname{char}(\mathbb{K}) \neq 2$, its branch curve is a plane quartic curve $B$ (see [13], Chapter V.6, page 67 ). Each curve $C_{i}$ is equal to the pre-image of a conic $K_{i}$ in the plane. Since $C_{i}$ is singular at three points $p_{j} \in \mathcal{Q} \backslash\left\{p_{i}\right\}$, the conic $K_{i}$ is tangent to $B$ at the images $q_{j}$ of the points $p_{j}$. In particular, the conics $K_{i}$ and $K_{j}$ are tangent to $B$ at two points. Consider the cubic curves $K_{i}+L_{i}$, where $L_{i}$ is the tangent line to $B$ at $q_{i}$. They all pass through $q_{1}, q_{2}, q_{3}$, and $q_{4}$ with tangent direction $L_{i}$ at $q_{i}$. Thus they generate a pencil of cubic curves with 8 base points (four are infinitely near the points $q_{i}$ ). The ninth base point must be the intersection point $q$ of the lines $L_{i}$. But then three tangents of the conic $K_{i}$ 
meet at $q$. This can happen only if $\operatorname{char}(\mathbb{K})=2$, so that $n=10$ and $X=Y$ when $\operatorname{char}(\mathbb{K}) \neq 2$.

Remark 5.8. The configuration of 4 conics with each pair tangent at two points is realized in characteristic 2. Consider the 3-dimensional linear system of conics $a x^{2}+b y^{2}+c z^{2}+d x y=0$. Each line through the point $(0: 0: 1)$ is tangent to all conics in the family. Choose four general points in the plane. For each subset of three of these points find a unique conic in the family which passes through these points. Then each pair of the four conics are tangent at two points.

Note that the pencil of cubic curves which we used in this proof defines a quasielliptic fibration on the blowup of the base points. It has 8 reducible fibers of type III in Kodaira's notation (two smooth rational curves tangent to each other at one point). There are no elliptic fibrations on a rational surface with such a combination of reducible fibers.

It remains to consider the case when $\operatorname{char}(\mathbb{K})=2$. The difference here is that the anticanonical linear system $\left|-K_{Z}\right|$ defines a separable map of degree 2 whose branch curve is a conic, and the condition that the pre-image of a conic is singular is not stated in terms of the tangency to the branch locus. So we have to find another argument.

Since at most 2 of the $\mathcal{P}_{j}$ are contained in cubic curves, we can assume that the $\mathcal{P}_{i}$ are not contained in cubic curves for $1 \leq i \leq 9$; each of these nine point sets is special, and is therefore a Coble set. Let $R_{i}$ be the proper transform of the sextic curve with double points at $\mathcal{P}_{i}, i=1, \ldots, 9$. The curves $R_{i}$ are pairwise disjoint $(-4)$-curves, and the divisor class of each $R_{i}$ is divisible by 2 . Let $D$ be the divisor $\sum R_{i}$, where $i$ runs from 1 to 9 , and let $\mathcal{L}$ be an invertible sheaf on $X$ such that $\mathcal{L}^{\otimes 2} \cong \mathcal{O}_{X}(D)$. This sheaf defines an inseparable double cover $\pi: Z \rightarrow X$ with branch divisor $D$. Recall that this means that $Z$ is locally isomorphic to Spec $\mathcal{O}_{X}(U)[T] /\left(T^{2}+\phi\right)$, where $U$ is an affine open set, $\phi$ is a local equation of $D$ in $U$, and $\pi_{*}\left(\mathcal{O}_{X}\right) \cong \mathcal{O}_{X} \oplus \mathcal{L}^{-1}$. Since $D$ is a reduced divisor, the cover has only finitely many singularities. The set of singularities supports the scheme of zeros of a section of $\Omega_{X}^{1} \otimes \mathcal{L}^{\otimes 2}$ (see [12, Proposition 0.1.2 7). The length of this 0-dimensional subscheme is equal to the second Chern class of the rank 2 locally free sheaf $\mathcal{E}=\Omega_{X}^{1} \otimes \mathcal{L}^{\otimes 2}$. The standard formula from the theory of Chern classes gives

$$
c_{2}(\mathcal{E})=c_{2}\left(\Omega_{X}^{1}\right)+c_{1}\left(\Omega_{X}^{1}\right) \cdot D+D^{2} .
$$

In our situation, we have $c_{2}\left(\Omega_{X}^{1}\right)=14$ (= the $l$-adic Euler characteristic of $\left.X\right)$ and $c_{1}\left(\Omega_{X}^{1}\right)=K_{X}$. This gives

$$
c_{2}(\mathcal{E})=14+K_{X} \cdot D+D^{2}=14+2 \times 9-4 \times 9=-4<0,
$$

a contradiction.

Thus, Theorem 5.1 is proved in all cases, including $\operatorname{char}(\mathbb{K})=2$.

\section{Cremona special Sets of $n \geq 10$ points lying on a Cubic Curve}

To establish our Main Theorem, it remains to prove Theorem 5.2, Assuming that $\mathcal{P}$ is Cremona special and lies on an irreducible cubic curve, we now prove

\footnotetext{
${ }^{7}$ This is analogous to the formula for the number of singular points of a holomorphic foliation on a complex surface $X$ defined by a section of $\Omega_{X}^{1} \otimes \mathcal{L}^{\otimes 2}$.
} 
that the set $\mathcal{P}$ is one of Harbourne's sets in this case. Note that, being Cremona special, $\mathcal{P}$ does not contain an infinitely near point.

\subsection{Torsion sets of points.}

Lemma 6.1. If $\mathcal{P}$ is a Cremona special set of points on a cubic curve $C_{0}$, then

(1) $C_{0}$ is irreducible;

(2) there exists a positive integer $s$ such that the divisor class of $s p_{i}$ in $\operatorname{Pic}\left(C_{0}\right)$ does not depend on the choice of $p_{i}$ in $\mathcal{P}$;

(3) $9 s p_{i} \in|3 s \mathfrak{h}|$ for all $p_{i} \in \mathcal{P}$, where $\mathfrak{h}=c_{1}\left(\mathcal{O}_{C_{0}}(1)\right)$, the divisor class of the intersection of $C_{0}$ with a line.

Proof of Lemma 6.1. We first assume that the number of points in $\mathcal{P}$ is equal to 10 . For all indices $i \in\{1, \ldots, 10\}$, denote by $\mathcal{P}_{i}$ the set $\mathcal{P} \backslash\left\{p_{i}\right\}$. Since $\mathcal{P}$ is Cremona special, so is $\mathcal{P}_{i}$. By Theorem 4.6, there exists a positive integer $m_{i}$ such that $\mathcal{P}_{i}$ is a Halphen set of index $m_{i}$. Since $\mathcal{P}_{i}$ is contained in $C_{0}$, this implies that $C_{0}$ is irreducible (otherwise the Halphen surface is not unnodal; hence $\mathcal{P}_{i}$ is not Cremona special), and that

$$
m_{i}\left(\sum_{j=1, j \neq i}^{10} p_{j}\right) \sim 3 m_{i} \mathfrak{h},
$$

where $\mathfrak{h}$ is the divisor obtained by intersecting $C_{0}$ with a line. Let $s$ be the least common multiple of the $m_{i}$, and let $\Sigma$ be the sum of the 10 points $p_{i}$. We obtain

$$
s\left(\Sigma-p_{i}\right) \sim 3 s \mathfrak{h} .
$$

Hence the divisor class of $s p_{i}$ does not depend on $p_{i}$. Summing up these equalities, we get $9 s \Sigma \sim 30 s \mathfrak{h}$, and thus $9 s p_{i} \sim 3 s \mathfrak{h}$.

If there are more than ten points in $\mathcal{P}$, we consider all subsets of ten points within $\mathcal{P}$. Properties (1), (2), and (3) hold for these subsets, and therefore also for $\mathcal{P}$ (for some positive integer $s$ ).

The following definition is due to Harbourne 24 .

Definition 6.2. A rational surface $X$ with Picard number $\rho(X) \geq 11$ and with $\left|-K_{X}\right|=\{D\}$ for some irreducible reduced curve $D$ is K3-like if the canonical restriction homomorphism

$$
\mathfrak{r}: K_{X}^{\perp} \rightarrow \operatorname{Pic}^{0}(D)
$$

has finite image.

We say that a point set $\mathcal{P}$ which is contained in an irreducible reduced cubic curve $C_{0}$ is a torsion set if the blow-up surface $X$ is K3-like. In that case, the strict transform $C$ of $C_{0}$ coincides with the unique member $D$ of the linear system $\left|-K_{X}\right|$.

When $\mathcal{P} \subset C_{0}$ is a torsion set, we denote by $X$ the surface obtained by blowing up $\mathcal{P}$, and by $S_{\mathfrak{r}}$ the kernel of the restriction homomorphism $\mathfrak{r}: K_{X}^{\perp} \rightarrow \operatorname{Pic}^{0}(C)$. The quotient group $K_{X}^{\perp} / S_{\mathfrak{r}}$ embeds as a finite subgroup in $\operatorname{Pic}^{0}(C)$; we denote by $m>1$ the smallest integer such that $K_{X}^{\frac{1}{X}} / S_{\mathfrak{r}}$ is contained in the $m$-torsion subgroup $\operatorname{Pic}^{0}\left(C_{0}\right)[m]$.

Corollary 6.3. A Cremona special set of $n \geq 10$ points on an irreducible cubic curve $C_{0}$ is a torsion set. 
Proof. The image of the restriction homomorphism $\mathfrak{r}: K_{X}^{\perp} \rightarrow \operatorname{Pic}^{0}\left(C_{0}\right)$ is generated by the divisor classes $\mathfrak{r}\left(e_{0}-e_{1}-e_{2}-e_{3}\right)$, and $\mathfrak{r}\left(e_{i}-e_{j}\right)$ for $i>j$. From Lemma 6.1, we have

$$
3 s \mathfrak{r}\left(e_{0}-e_{1}-e_{2}-e_{3}\right)=\mathfrak{r}\left(3 s \mathfrak{h}-9 s p_{1}\right)=0, s \mathfrak{r}\left(e_{i}-e_{j}\right)=\mathfrak{r}\left(s p_{i}-s p_{j}\right)=0 .
$$

This shows that the image of the restriction homomorphism is finitely generated and is contained in the $3 s$-torsion subgroup $\operatorname{Pic}\left(C_{0}\right)[3 s]$ of $\operatorname{Pic}^{0}(D)$. As such, $\mathfrak{r}\left(K_{X}^{\perp}\right)$ is finite.

Proposition 6.4. Let $\mathcal{P}$ be a torsion set of $n \geq 10$ points on an irreducible cubic curve $C_{0}$. Then $\mathcal{P}$ is Cremona special if and only if it is unnodal.

Proof. By Proposition 4.5, the condition that $\mathcal{P}$ is unnodal is necessary. By Theorem 3.2 from [24, it is also sufficient.

Let us sketch Harbourne's argument. Assume that $\mathcal{P}$ is unnodal. Since $\mathcal{P}$ is a torsion set, the surface $X$ is K3-like: The kernel $S_{\mathfrak{r}}$ or $\mathfrak{r}$ has finite index in $K_{X}^{\perp}$. By definition of $m, m K_{X}^{\perp} \subset S_{\mathfrak{r}}$. Let $w$ be an element of $W_{X}$. Let $E_{i} \subset X$ be the exceptional divisor obtained by blowing up $p_{i}$. The image of the class $e_{i}$ of $E_{i}$ by $w$ is represented by a unique $(-1)$-curve $E_{i}(w)$ (Lemma 3.4 assertion (i)). This curve intersects $C$ in a unique point $p_{i}(w)$ because $C \in\left|-K_{X}\right|$. Thus, $w$ transforms the point set $\mathcal{P}$ into a new point set $\left\{p_{1}(w), \ldots, p_{n}(w)\right\}$ of the curve $C_{0}$. This action is the same as the action of $W_{n}$ on the point sets, described in Section 1.5 (see also [20], §VI.5). On the other hand, the image of $\mathfrak{r}$ being finite, there exists a finite index subgroup $G$ of $W_{X}$ such that $\left\{p_{1}(w), \ldots, p_{n}(w)\right\}$ is projectively equivalent to $\mathcal{P}$ for all $w$ in $G$. From Section 1.5 (see [20], $\S \mathrm{VI}$ ), this implies that $G$ is realized as a subgroup of $\operatorname{Aut}(X)^{*}$; thus, $X$ is special.

Example 6.5 (Harbourne's examples; see Example 3.4 from 24]). Suppose that $\operatorname{char}(\mathbb{K})=p>0$ and $C_{0}$ is an irreducible cuspidal curve. The group $\operatorname{Pic}^{0}\left(C_{0}\right)$ is isomorphic to the abelian group $(\mathbb{K},+)$.

Let $C_{0}^{\#}$ be the complement of the singular point. Choose a point set $\mathcal{P}=$ $\left\{q_{1}, \ldots, q_{n}\right\}$ of $n \geq 10$ points on $C_{0}^{\#}$, denote by $X$ the blowup of $\mathcal{P}$ and consider the restriction homomorphism

$$
\mathfrak{r}: K_{X}^{\perp} \rightarrow \operatorname{Pic}^{0}\left(C_{0}\right) \cong \mathbb{K} .
$$

Its kernel $S_{\mathfrak{r}}$ is equal to $p K_{X}^{\frac{1}{X}}$ when the elements $3 \mathfrak{h}-q_{1}-q_{2}-q_{3}, q_{1}-q_{2}, \ldots$, and $q_{n-1}-q_{n}$ of $\operatorname{Pic}^{0}\left(C_{0}\right)$ are linearly independent over $\mathbb{F}_{p} \subset \mathbb{K}$; general point sets with $n \geq 10$ points satisfy this property.

Suppose, now, that $S_{\mathfrak{r}}=p K_{X}^{\frac{1}{X}}$. Since the divisor class of any $(-2)$-curve lies in the kernel of $\mathfrak{r}$ and, obviously, does not belong to $p K_{X}^{\perp}$, the point set $\mathcal{P}$ is unnodal. By Proposition 6.4, $\mathcal{P}$ is Cremona special.

From Proposition 6.4 we deduce that Theorem 5.2 is a consequence of the following statement.

Theorem 6.6. Let $C_{0} \subset \mathbb{P}^{2}$ be an irreducible cubic curve. Let $\mathcal{P} \subset C_{0}$ be a torsion set with $|\mathcal{P}|=10$, and let $m$ be the smallest integer such that the image of the restriction morphism $\mathfrak{r}$ is contained in $\mathrm{Pic}^{0}\left(C_{0}\right)[m]$. If $\mathcal{P}$ is unnodal, then $C_{0}$ is a cuspidal cubic and char $(\mathbb{K})$ divides $3 \mathrm{~m}$.

Note, in particular, that we assume in the following that the number $n$ of blowups is equal to 10 . Thus, $\mathcal{P}$ is now a torsion set of ten distinct points on an 
irreducible cubic curve $C_{0}$. As above, $C$ denotes the strict transform of $C_{0}$ in the surface $X$. The following lemmas provide a way to decide whether $\mathcal{P}$ is unnodal or not in terms of effective roots $\alpha \in K_{X}^{\perp}$ (see Section 3.3).

Lemma 6.7. Let $\mathcal{P}$ be a set of $n=10$ points on an irreducible cubic curve $C_{0}$. Let $\alpha \in K_{X}^{\perp}$ be an effective root. Any effective representative of $\alpha$ contains a $(-2)$-curve as one of its irreducible components.

Proof. Let $D$ be an effective representative of the root $\alpha$. Write $D$ as a positive sum $l C+\sum F_{i}$, where the $F_{i}$ are irreducible components different from $C$. Assume that no component $F_{i}$ is a $(-2)$-curve. Since $-K_{X}$ is represented by the irreducible curve $C$, we have $K_{X} \cdot F_{i} \leq 0$. The adjunction formula implies that any curve $F_{i}$ with negative self-intersection is a $(-1)$-curve. Since $\left(\sum F_{i}\right)^{2}=(D-l C)^{2}=-2-l^{2}<0$, some of the components $F_{i}$ are $(-1)$-curves. Write $\sum F_{i}=\mathcal{E}+\mathcal{A}$, where $\mathcal{E}$ is the sum of $(-1)$-components of $D$. We have

$$
\begin{gathered}
\mathcal{E}^{2} \leq \mathcal{E}^{2}+2 \mathcal{E} \cdot \mathcal{A}+\mathcal{A}^{2}=\left(\sum F_{i}\right)^{2}=-2-l^{2}, \\
\mathcal{E} \cdot C=(D-l C-\mathcal{A}) \cdot C=l-\mathcal{A} \cdot C \leq l .
\end{gathered}
$$

Write $\mathcal{E}=\sum k_{j} F_{j}$, where the curves $F_{j}$ in this sum are distinct $(-1)$-curves. The second inequality gives $\sum k_{j} \leq l$, and the first one gives $\sum k_{j}^{2} \geq 2+l^{2}$. This implies $2+l^{2} \leq \sum k_{j}^{2} \leq\left(\sum k_{j}\right)^{2} \leq l^{2}$, a contradiction.

It follows from this lemma that $X$ is unnodal if and only if it does not contain effective roots.

Lemma 6.8. Let $\mathcal{P}$ be a torsion set of $n=10$ points on an irreducible cubic curve $C_{0}$. The surface $X$ contains a $(-2)$-curve if and only if there exists a root $\alpha$ in the kernel $S_{\mathfrak{r}}$ of the restriction homomorphism $\mathfrak{r}: K_{X}^{\perp} \rightarrow \operatorname{Pic}^{0}(C)$.

Proof. Let $\alpha$ be a root. Consider the exact sequence

$$
0 \rightarrow \mathcal{O}_{X}\left(K_{X}+\alpha\right) \rightarrow \mathcal{O}_{X}(\alpha) \rightarrow \mathcal{O}_{C}(\alpha) \rightarrow 0,
$$

where the first nontrivial map is the multiplication by a section of $\mathcal{O}_{X}\left(-K_{X}\right)$ which vanishes along the curve $C$. The Riemann-Roch Formula, applied to the divisor class $-\alpha$, and Serre's Duality imply

$$
h^{0}(-\alpha)+h^{0}\left(K_{X}+\alpha\right)=h^{1}\left(K_{X}+\alpha\right) .
$$

Suppose $\alpha$ is the class of a $(-2)$-curve $R$. Since $C$ is an irreducible curve and $C \cdot R=0$, the curve $R$ is disjoint from $C$. Hence $\mathfrak{r}(\alpha)=\mathcal{O}_{C}(\alpha) \cong \mathcal{O}_{C}$, and thus $\mathfrak{r}(\alpha)=0$ in $\operatorname{Pic}(C)$. Conversely, suppose $\mathcal{O}_{C}(\alpha) \cong \mathcal{O}_{C}$. Then $h^{0}\left(\mathcal{O}_{C}(\alpha)\right) \neq 0$; hence either $h^{0}\left(\mathcal{O}_{X}(\alpha)\right) \neq 0$, or $h^{1}\left(\mathcal{O}_{X}\left(K_{X}+\alpha\right)\right) \neq 0$. In the second case,

$$
0<h^{1}\left(K_{X}+\alpha\right)=h^{0}\left(K_{X}+\alpha\right)+h^{0}(-\alpha) \leq h^{0}(\alpha)+h^{0}(-\alpha)
$$

implies that $\alpha$ or $-\alpha$ is effective. Thus, in both cases, either $\alpha$ or $-\alpha$ is an effective root and Lemma 6.7 implies that $X$ contains a $(-2)$-curve.

\subsection{The reduction to a question on the arithmetic of quadratic forms.}

The proof of Theorem 6.6 that we now describe is rather delicate, and uses strong approximation results in a specific situation; unfortunately, we have not been able to find a simpler geometric argument.

We assume that $\mathcal{P} \subset C_{0}$ is a torsion set and make use of the notation introduced in the previous section. We assume that either (i) $C_{0}$ is not cuspidal or (ii) $C_{0}$ is 
cuspidal but char $(\mathbb{K})$ does not divide $3 m$, and our goal is to show that $X$ contains a $(-2)$-curve; by Lemma 6.8, all we need to prove is the existence of a root $\alpha$ in the kernel $S_{\mathfrak{r}}$ of the morphism $\mathfrak{r}$.

Note that, under our assumptions, $\operatorname{Pic}^{0}(C)[m] \cong(\mathbb{Z} / m Z)^{a}$, where $a \leq 2$. Obviously, $S_{\mathfrak{r}}$ contains the sublattice $m K_{X}^{\perp}$. Define $V_{m}=S_{\mathfrak{r}} / m K_{X}^{\perp}$ and $L_{m}=$ $K_{X}^{\perp} / m K_{X}^{\perp} ; V_{m}$ is a submodule of $L_{m}$ over the $\operatorname{ring} \mathbb{Z} / m \mathbb{Z}$ :

$$
V_{m}=S_{\mathfrak{r}} / m K_{X}^{\perp} \subset L_{m}=K_{X}^{\perp} / m K_{X}^{\perp} \cong(\mathbb{Z} / m \mathbb{Z})^{10} .
$$

This submodule $V_{m}$ contains a free $(\mathbb{Z} / m \mathbb{Z})$-submodule of dimension 8 because $a \leq 2$. The morphism $\mathfrak{r}$ induces a morphism from $L_{m}$ to $\operatorname{Pic}^{0}(C)[m]$, and Theorem 6.6 becomes a consequence of the following purely arithmetic statement.

Theorem 6.9. Let $m$ be a positive integer. Let $L_{m}=\mathbb{E}_{10} / m \mathbb{E}_{10}$ and $V_{m}$ be a $(\mathbb{Z} / m \mathbb{Z})$-submodule of $L_{m}$ containing a free submodule of rank 8 . Then there exists a root in $\mathbb{E}_{10}$ whose projection into $L_{m}$ is contained in $V_{m}$.

We employ the theory of quadratic forms over any commutative ring $A$. Let $M$ be a finitely generated $A$-module. A function $q: M \rightarrow A$ is called a quadratic form if

- for any $x \in M$ and $a \in A, q(a x)=a^{2} q(x)$;

- $b_{q}(x, y)=q(x+y)-q(x)-q(y)$ is a symmetric bilinear map $M \times M \rightarrow A$.

The bilinear form $b_{q}$ is called the associated symmetric bilinear form. If 2 is invertible in $A$, then $q=2^{-1} b(x, x)$, and the notion of a quadratic form is equivalent to the notion of a symmetric bilinear form. A module $M$ equipped with a quadratic form $q$ is called a quadratic module.

We denote by b the bilinear form on $K_{X}^{\perp}$ given by the intersection product, and we equip $L_{m}$ with the symmetric bilinear form $\mathrm{b}_{m}$ obtained by reduction of $\mathrm{b}$ modulo $m$. When $m$ is even, we equip $L_{m}$ with the structure of a quadratic module by setting

$$
q_{m}(x)=\frac{1}{2} \mathrm{~b}_{m}(x, x)
$$

(recall that our lattice $K_{X}^{\frac{1}{X}}$ is an even unimodular lattice). Let $\mathrm{O}\left(L_{m}\right)$ be the orthogonal group of $\left(L_{m}, \mathrm{~b}_{m}\right)$ (resp. of $\left(L_{m}, q_{m}\right)$ when $m$ is even).

To prove Theorem 6.9, fix a root $\alpha$ and consider its image $\bar{\alpha}$ in $L_{m}$. Suppose we find an element $\sigma \in \mathrm{O}\left(L_{m}\right)$ such that $\sigma(\bar{\alpha}) \in V_{m}$. Suppose, moreover, that $\sigma$ lifts to an element $w \in \mathrm{O}\left(K_{X}^{\perp}\right)$. Then $w(\alpha)$ is a root, $w(\alpha)$ is contained in $S_{\mathfrak{r}}$, and we are done. We now develop this strategy.

6.3. Orthogonal and Spin groups modulo $p^{l}$. Since the quadratic form $\mathrm{b}$ of the lattice $\mathbb{E}_{10}$ is unimodular, there is a connected smooth group scheme $\mathbf{S O}_{\mathrm{b}}$ over $\mathbb{Z}$ such that $\mathbf{S O}_{\mathrm{b}}(\mathbb{Z})$ coincides with the group of isometries of the lattice $\mathbb{E}_{10}$ with determinant 1 .

The universal cover of $\mathbf{S O}_{\mathrm{b}}$ is a smooth group scheme $\mathbf{S p i n}_{\mathrm{b}}$ over $\mathbb{Z}$; the group $\operatorname{Spin}_{b}(\mathbb{Z})$ is the group $\operatorname{Spin}\left(\mathbb{E}_{10}\right)$ of all invertible elements of the even part of the Clifford algebra of the quadratic $\mathbb{Z}$-module $\mathbb{E}_{10}$ such that the corresponding inner automorphism leaves $\mathbb{E}_{10}$ invariant (see [29], Chapter IV, §5).

There is an exact sequence of group schemes

$$
1 \rightarrow \boldsymbol{\mu}_{2} \rightarrow \mathbf{S p i n}_{\mathrm{b}} \rightarrow \mathbf{S O}_{\mathrm{b}} \rightarrow 1,
$$


where $\boldsymbol{\mu}_{2}$ is the group of square roots of 1 . For any commutative ring $A$ with $\operatorname{Pic}(A)=0$, the exact sequence defines the following exact sequence of groups:

$$
1 \rightarrow \boldsymbol{\mu}_{2}(A) \rightarrow \operatorname{Spin}_{\mathrm{b}}(A) \rightarrow \mathbf{S O}_{\mathrm{b}}(A) \rightarrow A^{*} / A^{* 2}
$$

(see [29], Theorem 6.2.6). This will be applied to $A=\mathbb{Z} / p^{k} \mathbb{Z}$ for prime numbers $p$. In this case $\left|A^{*} / A^{* 2}\right|=\left|\boldsymbol{\mu}_{2}(A)\right|$; this number is equal to 2 if $p \neq 2$, and to 1,2 , or 4 if $p=2$ and $k=1, k=2$, or $k \geq 3$ respectively. Thus the image of $\operatorname{Spin}_{\mathrm{b}}(A)$ in $\mathbf{S O}_{\mathrm{b}}(A)$ is of index at most 4 .

The group scheme $\mathbf{S p i n}_{\mathrm{b}}$ is requested in order to apply the Strong Approximation Theorem (see [28, Theorem 24.6):

Theorem 6.10 (Strong Approximation). Let $M$ be a unimodular indefinite integral quadratic lattice of rank $\geq 3$; if $p$ is a prime integer, denote by $M_{p}=M \otimes \mathbb{Z}_{p}$ its $p$-adic localization at $p$. Let $p_{i}, i \in I$, be a finite set of prime numbers. Then the canonical homomorphism

$$
\operatorname{Spin}(M) \rightarrow \prod_{i \in I} \operatorname{Spin}\left(M_{p_{i}}\right)
$$

has a dense image.

Note that, by the Chinese Remainder Theorem,

$$
\mathrm{O}\left(L_{m}\right)=\prod_{i} \mathrm{O}\left(L_{p_{i}^{k_{i}}}\right)
$$

where $m=\prod p_{i}^{k_{i}}$ is the prime factorization of $m$.

Since the fibers of the natural homomorphism $\operatorname{Spin}\left(M_{p_{i}}\right) \rightarrow \operatorname{Spin}\left(M / p_{i}{ }^{k_{i}}\right)$ are open subsets of $\operatorname{Spin}\left(M_{p_{i}}\right)$, we obtain a commutative diagram

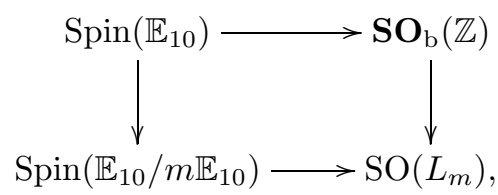

for which the first vertical arrow is surjective, by the Strong Approximation Theorem. Suppose we find a root $\alpha \in \mathbb{E}_{10}$ and an element $\sigma$ in the image of the bottom horizontal arrow such that $\sigma(\bar{\alpha}) \in V_{m}$. Then, the commutative diagram shows that $\sigma$ can be lifted to an element $w \in \mathbf{S O}_{\mathrm{b}}(\mathbb{Z})$ such that the projection of $w(\alpha)$ into $L_{m}$ is contained in $V_{m}$, and therefore Theorem 6.9 is proved.

6.4. Orbits of $\mathrm{SO}_{\mathrm{b}}$ and $\mathrm{Spin}_{\mathrm{b}}$ modulo $p$. This section is a warm-up for the following ones. Fix a root $\alpha$ in $\mathbb{E}_{10}$ and denote by $\bar{\alpha}$ its image in $L_{p}$, where $p$ is a prime integer.

Since $L_{p}$ is a nondegenerate quadratic space over $\mathbb{F}_{p}$, the rank of any submodule of $L_{p}$ on which the quadratic form vanishes identically modulo $p$ is at most 5 . Any quadratic form of rank $\geq 2$ over $\mathbb{F}_{p}$ represents all elements in $\mathbb{F}_{p}^{*}$ ([39], Chapter I). Thus, $b_{p}$ represents all elements of $\mathbb{F}_{p}^{*}$.

Let $r \in V_{p}$ be an element with $r^{2}:=b_{p}(r, r)=-2$. If $p \neq 2$, Witt's Theorem provides an element $\sigma \in \mathrm{O}\left(L_{p}\right)$ such that $\sigma(\bar{\alpha})=r$.

If $p=2$, we consider $L_{2}=\mathbb{E}_{10} / 2 \mathbb{E}_{10} \cong \mathbb{F}_{2}^{10}$ as a quadratic space with the quadratic form $q_{2}(x)=\frac{1}{2} b(x, x) \bmod 2$. Since $V_{2}$ is of dimension $\geq 8, q_{2}$ does not vanish identically on $V_{2}$. There are $496=2^{4}\left(2^{5}-1\right)$ vectors in the set $q_{2}^{-1}(1)$ and 
all of them are represented by roots (see [11], Remark 4.7). Thus there are elements of $V_{2}$ which are represented by roots.

As a consequence, there is a root $\alpha$ in $\mathbb{E}_{10}$, such that, for each prime number $p$, there is an element $\sigma \in \mathrm{O}\left(L_{p}\right)$ satisfying $\sigma(\bar{\alpha}) \in V_{p}$.

We now extend this idea to the case $m=p^{k}$ with $k>1$, and use this extension to prove Theorem 6.9.

6.5. Quadratic modules and orbits modulo $p^{k}$. Here, the ring is $A=\mathbb{Z} / p^{k} \mathbb{Z}$ and the quadratic module is $M=V_{m}$, equipped with the symmetric bilinear form $b_{p^{k}}$ or the quadratic form $q_{p^{k}}$ when $p=2$. Since $A$ is local, with maximal ideal $\mathfrak{m}=p A$, an element $a$ in $A$ is invertible if and only if $a$ is not contained in $\mathfrak{m}$.

Lemma 6.11. The quadratic module $V_{p^{k}}$ represents all invertible elements of the ring $\mathbb{Z} / p^{k} \mathbb{Z}$ (i.e. $\forall a \in \mathbb{Z} / p^{k} \mathbb{Z}$, there exists $x \in V_{p^{k}}$ such that $q(x)=a$ ). The same property holds for all free quadratic submodules $M_{0} \subset V_{p^{k}}$ of rank 8 .

Proof. We prove the lemma for $V_{p^{k}}$ by induction on $k$. The same proof applies for submodules of $V_{p^{k}}$ of rank 8.

When $k=1, V_{p}$ represents all nonzero elements of the field $\mathbb{F}_{p}$, as explained in Section 6.4. More precisely, for all $a \in \mathbb{F}_{p}^{*}$ there exists a pair of vectors $(v, w)$ in $V_{p}$ such that $q(v)=a \bmod p$ and $b_{q}(v, w)=1 \bmod p$.

Let $k$ be a positive integer. The induction hypothesis asserts that for all invertible elements $a$ of $\mathbb{Z} / p^{k} \mathbb{Z}$ there exists a pair of vectors $(v, w)$ in $V_{p^{k}}$ such that

$$
q(v)=a \bmod p^{k} \quad \text { and } \quad b_{q}(v, w)=1 \quad \bmod p^{k} .
$$

Let $a$ be an invertible element of $\mathbb{Z} / p^{k+1} \mathbb{Z}$. Apply the induction hypothesis to find elements $v$ and $w$ of $V_{p^{k+1}}$ such that $q(v)=a \bmod p^{k}$ and $b_{q}(v, w)=1 \bmod p^{k}$. Then $b_{q}(v, w)$ is invertible in $\mathbb{Z} / p^{k+1} \mathbb{Z}$ and changing $w$ in one of its multiples we construct a vector $w$ such that $b_{q}(v, w)=1 \bmod p^{k+1}$. Write $q(v)=a+b p^{k}$ and change $v$ into $v^{\prime}=v-b p^{k} w$; then $q\left(v^{\prime}\right)=a \bmod p^{k+1}$. We still have $b_{q}\left(v^{\prime}, w\right)=1$ $\bmod p^{k}$, so that a multiple $w^{\prime}$ of $w$ satisfies $b_{q}\left(v^{\prime}, w^{\prime}\right)=1$. The lemma is proved by induction.

Now we invoke the following analog of Witt's Theorem for quadratic modules over local rings (see [28], (4.4)).

Lemma 6.12 (Witt's Theorem). Let $M$ be a quadratic module over a local ring $A$ with maximal ideal $\mathfrak{m}$. Let $F, G$ be free primitive submodules of $M$ over the ring $A$. Any isomorphism of quadratic modules $F \rightarrow G$ extends to an automorphism of the quadratic module $M$.

Here primitive means that the quotient module is free.

6.6. Proof of Theorem 6.9. We are ready to prove Theorem 6.9, hence Theorem 6.6. and Theorem 5.2.

Let $m$ be a positive integer and $\prod_{i} p_{i}^{k_{i}}$ be its decomposition into prime factors. Let $\alpha \in \mathbb{E}_{10}$ be a root (for example $\alpha=e_{1}-e_{2}$ ).

Let $p^{k}$ be any of the factors $p_{i}^{k_{i}}$. Consider the image $\bar{\alpha}$ of $\alpha$ in $L_{p^{k}}$. Fix a free submodule $M_{0}$ of rank 8 in $V_{p^{k}}$. By Lemma 6.11, there is an element $v \in M_{0}$ with $q_{p^{k}}(v)=-2 \in \mathbb{Z} / p^{k} \mathbb{Z}$ if $p \neq 2$ and $q_{p^{k}}(v)=1$ if $p=2$. The element $v$ generates a free primitive submodule of $L_{p^{k}}$. By Witt's Theorem, we find an 
element $\sigma \in \mathrm{O}\left(L_{p^{k}}\right)$ such that $\sigma(\bar{\alpha})=v$; in particular, $\sigma(\bar{\alpha})$ is contained in $V_{p^{k}}$. Let us show that $\sigma$ can be chosen in the image of the map $\operatorname{Spin}\left(L_{p^{k}}\right) \rightarrow \mathrm{O}\left(L_{p^{k}}\right)$.

Recall that the reflection $s_{h}$ with respect to a vector $h$, for which $q(h)$ is invertible, is defined by the formula

$$
s_{h}(x)=x-\frac{b(x, h)}{q(h)} h .
$$

If $h$ is in $M_{0}, s_{h}$ is an isometry of $L_{p^{k}}$ that preserves $M_{0}$. By Theorem (4.6) from [28], any isometry of $M_{0}$ (resp. $L_{p^{k}}$ ) is the product of reflections in elements from $M_{0}$ (resp. $\left.L_{p^{k}}\right)$. As explained in [28, page 39, Section 8, an element $\eta$ of $\mathrm{O}\left(L_{p^{k}}\right)$ is in the image of $\operatorname{Spin}\left(L_{p^{k}}\right)$ if and only if $\eta$ is a product of an even number of reflections, $\eta=s_{h_{1}} \circ \cdots \circ s_{h_{2 s}}$, and its spinor $\operatorname{norm} \operatorname{SN}(\eta)$ is 1, i.e.

$$
\operatorname{SN}(\eta):=q\left(h_{1}\right) \cdots q\left(h_{2 s}\right)=1 \quad \bmod \left(\mathbb{Z}_{p^{k}}^{*}\right)^{2} .
$$

(See also [29], chapter IV, §6.)

Write $\sigma$ as a composition of reflections $s_{h_{i}}, 1 \leq i \leq l$, with $q\left(h_{i}\right) \in \mathbb{Z}_{p^{k}}^{*}$ ( $l$ may be odd). Apply Lemma 6.11 to find vectors $h_{l+1}$ and $h_{l+2}$ in $M_{0}$ such that

$$
q\left(h_{l+1}\right)=\prod_{i=1}^{l} q\left(h_{i}\right)^{-1} \quad \text { and } \quad q\left(h_{l+2}\right)=1 \quad \bmod \left(\mathbb{Z}_{p^{k}}^{*}\right)^{2} .
$$

Change $\sigma$ into $s_{h_{l+1}} \circ \sigma$ or $s_{h_{l+2}} \circ s_{h_{l+1}} \circ \sigma$ to obtain an isometry which is a product of an even number of reflections. After such a modification, $\operatorname{sN}(\sigma)=1$ and $\sigma$ is in the image of $\operatorname{Spin}\left(L_{p^{k}}\right)$. Since $M_{0}$ is preserved by $s_{h_{l+1}}$ and $s_{h_{l+2}}, \sigma(\bar{\alpha})$ is contained in $M_{0}$, and thus in $V_{p^{k}}$.

Now let $\bar{\alpha}$ denote the image of $\alpha$ into $L_{m}$. Since the previous argument applies to all prime factors $p_{i}^{k_{i}}$ of $m$, the Chinese Remainder Theorem shows the existence of an element $\sigma$ in $\operatorname{Spin}\left(\mathbb{E}_{10} / m \mathbb{E}_{10}\right)$ such that $\sigma(\bar{\alpha}) \in V_{m}$. By the Strong Approximation Theorem, $\sigma$ lifts to an element $\sigma^{\prime}$ in $\operatorname{Spin}\left(\mathbb{E}_{10}\right)$; then, the image $w$ of $\sigma^{\prime}$ in $\operatorname{SO}_{\mathrm{b}}(\mathbb{Z})$ maps the root $\alpha$ onto a root $w(\alpha)$ whose projection modulo $m$ is in $V_{m}$. This proves Theorem 6.9 and hence Theorem 6.6

\section{NON-ALGEBRAICALLY CLOSED FIELDS AND OTHER SURFACES}

7.1. Non-algebraically closed fields. In this section, the ground field $\mathbb{K}$ is not necessarily algebraically closed. Let $\overline{\mathbb{K}}$ be its algebraic closure and let $\bar{X}=X \otimes_{\mathbb{K}} \overline{\mathbb{K}}$ be obtained from $X$ by base field change. Let $W_{\bar{X}}$ denote the Coxeter subgroup of $\mathrm{O}\left(\operatorname{Pic}\left(X_{\overline{\mathbb{K}}}\right)\right)$, as defined in Section 1.3 . We have a sequence of inclusions

$$
\operatorname{Aut}(X)^{*} \subset \operatorname{Aut}(\bar{X})^{*} \subset W_{\bar{X}} \subset \mathrm{O}\left(K_{\bar{X}}^{\perp}\right) \text {. }
$$

We say that $X$ is Cremona special over $\mathbb{K}$ if $\operatorname{Aut}(X)^{*}$ has finite index in $W_{\bar{X}}$ and $W_{\bar{X}}$ is infinite. The following result extends the Main Theorem to arbitrary fields $\mathbb{K}$.

Theorem 7.1. If $X$ is Cremona special over $\mathbb{K}$, then

- $\bar{X}$ is unnodal;

- $X$ is obtained from $\mathbb{P}_{\mathbb{K}}^{2}$ by blowing up a finite subset of $\mathbb{P}^{2}(\mathbb{K})$;

- $X$ is a Halphen, a Coble, or a Harbourne example (over $\mathbb{K}$ ).

Proof. Let $n+1$ be the Picard number of $\bar{X}$. Assume that $X$ is Cremona special over $\mathbb{K}$; in particular, $X$ is obtained from $\mathbb{P}_{\mathbb{K}}^{2}$ by blowing up a 0 -cycle $\mathcal{P}$ of length $n$ defined over $\mathbb{K}$. Since $\operatorname{Aut}(\bar{X})$ contains $\operatorname{Aut}(X), \bar{X}$ is Cremona special. 
From our Main Theorem, we deduce that $\bar{X}$ is unnodal, $\mathcal{P}$ is made of $n$ distinct points of $\mathbb{P}^{2}(\overline{\mathbb{K}})$, and $\bar{X}$ is a Halphen, a Coble, or a Harbourne example over $\mathbb{K}$. In the Halphen case, the genus 1 fibration on $X$ is unique. In the Coble case, $\left|-2 K_{\bar{X}}\right|$ contains a unique member (the strict transform of the sextic curve with double points along $\mathcal{P}$ ). In the Harbourne case, when the Picard number of $\bar{X}$ is $\geq 10,\left|-K_{\bar{X}}\right|$ contains also a unique member (given by the proper transform of the cuspidal cubic containing $\mathcal{P}$ ). Thus, these curves and pencil are defined over $\mathbb{K}$.

Let us show that $\operatorname{Pic}(X)$ has finite index in $\operatorname{Pic}(\bar{X})$. First, assume that $n \geq$ 10. Consider the subgroup $\operatorname{Pic}(X)$ of $\operatorname{Pic}(\bar{X})$. It contains ample classes and the canonical class $K_{X}$; in particular, it intersects $K_{\bar{X}}^{\perp}$ on an infinite subgroup $L$ which is $\operatorname{Aut}(X)^{*}$-invariant. But $\operatorname{Aut}(X)^{*}$ has finite index in $W_{\bar{X}}, W_{\bar{X}}$ is Zariski dense in $\mathrm{O}\left(K_{X}^{\perp} \otimes \mathbb{R}\right)$, and the action of this orthogonal group on $K_{X}^{\perp} \otimes \mathbb{R}$ is irreducible. Thus, $L \otimes \mathbb{R}$ coincides with $K_{X}^{\perp} \otimes \mathbb{R}$. Since $\operatorname{Pic}(X)$ also contains $K_{X}$, we deduce that $\operatorname{Pic}(X) \otimes \mathbb{R}$ is equal to $\operatorname{Pic}(\bar{X}) \otimes \mathbb{R}$ and that $\operatorname{Pic}(X)$ has finite index in $\operatorname{Pic}(\bar{X})$.

When $n=9$, one needs a slightly different argument. Since $\operatorname{Pic}(X)$ contains ample classes, the intersection form restricts to a form of signature $(1, m)$ on $\operatorname{Pic}(X)$, with $m \leq 9$. In particular, the intersection form is negative definite on the orthogonal complement of $\operatorname{Pic}(X)$, and the action of $\operatorname{Aut}(X)$ on $\operatorname{Pic}(X)$ has finite kernel. Since the action of $\operatorname{Aut}(X)$ on $\operatorname{Pic}(X)$ preserves the isotropic vector $K_{X}$ and the integral structure, it contains a finite index, free abelian subgroup of rank at most $m-1$. Since $\operatorname{Aut}(X)^{*}$ has finite index in $W_{9}$, we deduce that $m=9$ because $W_{9}$ contains a free abelian group of rank 8 . This shows that $\operatorname{Pic}(X)$ has finite index in $\operatorname{Pic}(\bar{X})$.

Let us now prove that all points $p_{i}$ of $\mathcal{P}, 1 \leq i \leq n$, are in fact defined over $\mathbb{K}$, i.e. that $\mathcal{P} \subset \mathbb{P}^{2}(\mathbb{K})$. It suffices to show that all $(-1)$-curves $E$ in $\bar{X}$ are defined over $\mathbb{K}$. Since $\operatorname{Pic}(X)$ has finite index in $\operatorname{Pic}(\bar{X})$, the divisor class of some positive multiple $m E$ is in $\operatorname{Pic}(X)$. Since $|m E|=\{m E\}$, we obtain that $m E$ is defined over $\mathbb{K}$ and hence $E$ is defined over $\mathbb{K}$.

7.2. Other types of surfaces. We can extend the concept of Cremona special rational surfaces to other types of projective surfaces as follows. One says that a surface $Y$ has a large automorphism group if $\operatorname{Aut}(Y)^{*}$ is infinite and of finite index in the orthogonal group $\mathrm{O}\left(K_{Y}^{\perp}\right) \subset \mathrm{O}(\operatorname{Num}(Y))$, where $\operatorname{Num}(Y)$ is the lattice of divisor classes modulo numerical equivalence. Besides rational surfaces, other candidates of surfaces with large automorphism groups are surfaces of Kodaira dimension 0 or 1 . Indeed, $\operatorname{Aut}(Y)$ is finite if the Kodaira dimension of $Y$ is 2 , and $\operatorname{Aut}(Y)^{*}$ is finite if $Y$ is ruled but not rational.

7.2.1. Kodaira dimension 1. If the Kodaira dimension of $Y$ is 1 , some multiple of the canonical class defines an elliptic (or quasi-elliptic) fibration on $Y$. The Mordell-Weil group of the corresponding Jacobian fibration embeds as a finite index subgroup into $\operatorname{Aut}(Y)^{*}$. By the Shioda-Tate formula, the rank of this group is equal to $\rho(Y)-2$, provided there are no reducible fibers in the genus 1 fibration. Using the argument from the proof of Theorem 2.10, one can show, in this case, that $\operatorname{Aut}(Y)^{*}$ is of finite index in $\mathrm{O}\left(K_{Y}^{\perp}\right)$. Thus, $\operatorname{Aut}(Y)^{*}$ has finite index in $\mathrm{O}\left(K_{Y}^{\perp}\right)$ if and only if the canonical fibration has no reducible fiber.

7.2.2. Kodaira dimension 0 . The classification of surfaces implies that minimal surfaces with Kodaira dimension 0 fall into four types: Abelian surfaces, K3 surfaces, Enriques surfaces, and bielliptic surfaces. 
Theorem 7.2. Let $\mathbb{K}$ be an algebraically closed field with $\operatorname{char}(\mathbb{K}) \neq 2$. Let $Y$ be a projective surface over $\mathbb{K}$ with Kodaira dimension equal to 0 . If $\operatorname{Aut}(Y)$ is large, then $Y$ is minimal and $Y$ is not a bielliptic surface. If $Y$ is an abelian surface, a $K 3$ surface, or an Enriques surface, then $\operatorname{Aut}(Y)$ is large if and only if $\mathrm{O}(\mathrm{Num}(Y))$ is infinite and $Y$ does not contain any smooth rational curve.

Sketch of the proof. Recall that a $(-k)$-curve is a smooth rational curve with selfintersection $-k$. Let $Y$ be a projective surface with Kodaira dimension 0 . The set of classes of $(-1)$-curves is finite, and permuted by $\operatorname{Aut}(Y)$; thus, if $\operatorname{Aut}(Y)$ is large, then $Y$ is minimal and $K_{Y}^{\perp}=\operatorname{Num}(Y)$. If $Y$ is bielliptic, it is easily checked that $\operatorname{Aut}(Y)^{*}$ is finite.

Abelian surfaces do not contain rational curves, and smooth rational curves on K3 surfaces and Enriques surfaces are $(-2)$-curves. Each of them defines a reflection on $\operatorname{Num}(Y)$. Denote by $\operatorname{Nod}(Y)$ the set of smooth rational curves and by $\operatorname{Ref}(Y) \subset \mathrm{O}(\operatorname{Num}(Y))$ the group generated by the reflections around classes of smooth rational curves.

Let $Y$ be an abelian or K3 surface. Over the field of complex numbers, the Torelli Theorem implies that $\operatorname{Aut}(Y)^{*}$ is of finite index in $\mathrm{O}(\operatorname{Num}(Y))$ if and only if $\operatorname{Nod}(Y)$ is empty (this is always true for abelian surfaces). For K3 surfaces this fact has been extended recently to any characteristic $p \neq 2$ by M. Lieblich and D. Maulik (see [30]). They also extended another corollary of the Torelli Theorem: The set of orbits of smooth rational curves with respect to the automorphism group is finite. Their arguments can probably be adapted to abelian surfaces. Thus, an abelian surface or K3 surface has a large automorphism group if and only if $\mathrm{O}(\mathrm{Num}(Y))$ is infinite and $\operatorname{Nod}(Y)$ is empty 8

Finally, the automorphism group of an Enriques surface without smooth rational curves is always large, and this is true in any characteristic. The proof and the statement are analogous to the proof of Theorem 3.5 (see [15], for $\mathbb{K}=\mathbb{C}$ ).

7.2.3. Complex surfaces and rational curves. Let us assume that $Y$ is a complex projective surface.

(1) If $Y$ is an abelian surface, $Y$ does not contain rational curves;

(2) if $Y$ is a K3 surface and $Y$ does not contain any smooth rational curve, its Picard number satisfies $\rho(Y) \leq 11$;

(3) a generic Enriques surface contains no smooth rational curve.

The first assertion is easily proved, and the third is contained in [2. The second one was explained to the authors by V. Nikulin: Any $K 3$ surface with $\rho(Y) \geq 12$ contains a smooth rational curve (see [38, Theorems 14 and 15).

To prove it, assume $\rho(Y) \geq 12$. Since $H^{2}(Y, Z)$ is an even unimodular lattice of dimension 22 and signature $(3,19)$, the orthogonal complement $T(Y)$ of $\operatorname{Num}(Y)$ in $H^{2}(Y, Z)$ has dimension $\leq 10$. By Theorem 1.13.1* (or 1.13.2) in [36], the primitive embedding $T(Y) \subset H^{2}(Y, Z)$ is unique up to an isomorphism, because $\operatorname{Num}(U)$ is indefinite and $\operatorname{rank}(T(Y)) \leq 10$ (see also Corollaries 2.9 and 2.10 in [32]). On the other hand, since $\operatorname{rank}(T(Y)) \leq 10$, Theorem 1.12 .2 of $[36$ implies the existence of a primitive embedding

$$
T(Y) \oplus\langle-2\rangle \subset H^{2}(Y, Z)
$$

\footnotetext{
${ }^{8}$ If $\operatorname{Aut}(Y)^{*}$ is infinite and $Y$ contains smooth rational curves, then $\operatorname{Ref}(Y)$ is infinite and its elements represent different cosets of $\mathrm{O}(\operatorname{Num}(Y))$ modulo $\operatorname{Aut}(Y)^{*}$.
} 
where $\langle-2\rangle$ denotes the 1-dimensional lattice generated by a vector with selfintersection -2 . Taking the orthogonal complement for this second embedding, one obtains that $\operatorname{Num}(Y)$ contains a copy of the sublattice $\langle-2\rangle$.

This shows that K3 surfaces with large automorphism groups must satisfy $\rho(Y)$ $\leq 11$. This is a strange coincidence with our main result that complex rational surfaces with large automorphism groups must satisfy $\rho=10$ or 11 .

Corollary 7.3. Let $Y$ be a complex projective surface with large automorphism group. If $\operatorname{Aut}(Y)^{*}$ does not contain any finite index abelian group, the Picard number of $Y$ is at most 11.

Indeed, the assumption implies that $\operatorname{Aut}(Y)^{*}$ is infinite but does not preserve a genus 1 fibration, since otherwise the Mordell-Weil group of the corresponding Jacobian fibration would determine a finite index abelian subgroup of $\operatorname{Aut}(Y)^{*}$. Thus, either $Y$ is rational, or its Kodaira dimension vanishes. The conclusion follows from the Main Theorem, [7.2, and Nikulin's argument.

\section{ACKNOWLEDGEMENT}

The authors thank A. Chambert-Loir, J.-L. Colliot-Thélène, Y. de Cornulier, M. W. Davis, M. H. Gizatullin, D. Harari, J. Keum, S. Kondō, C.T. McMullen, V. Nikulin, and G. Prasad for interesting discussions on the topics of this paper. The authors also thank the thorough referees for their careful reading and their comments which allowed them to clarify the exposition and to correct some of the arguments of the previous version of this paper.

\section{REFERENCES}

[1] Wolf P. Barth, Klaus Hulek, Chris A. M. Peters, and Antonius Van de Ven. Compact complex surfaces, volume 4 of Ergebnisse der Mathematik und ihrer Grenzgebiete. Springer-Verlag, Berlin, second edition, 2004. MR2030225 (2004m:14070)

[2] Wolf P. Barth and Chris A. M. Peters. Automorphisms of Enriques surfaces. Invent. Math., 73(3):383-411, 1983. MR718937 (85g:14052)

[3] Eric Bedford and Kyounghee Kim. Periodicities in linear fractional recurrences: degree growth of birational surface maps. Michigan Math. J., 54(3):647-670, 2006. MR2280499 (2008k:32054)

[4] Yves Benoist and Pierre de la Harpe. Adhérence de Zariski des groupes de Coxeter. Compos. Math., 140(5):1357-1366, 2004. MR2081159 (2005g:20059)

[5] Serge Cantat. Dynamique des automorphismes des surfaces K3. Acta Math., 187(1):1-57, 2001. MR:1864630 (2003h:32026)

[6] Serge Cantat. Sur la dynamique du groupe d'automorphismes des surfaces K3. Transform. Groups, 6(3):201-214, 2001. MR1854708 (2002k:37039)

[7] Serge Cantat. Bers and Hénon, Painlevé and Schrödinger. Duke Math. J., 149(3):411-460, 2009. MR2553877 (2011f:37077)

[8] Serge Cantat and Charles Favre. Symétries birationnelles des surfaces feuilletées. J. Reine Angew. Math., 561:199-235, 2003. MR1998612(2004i:32048)

[9] Arthur B. Coble. The ten nodes of the rational sextic and of the Cayley symmetroid. Amer. J. Math., 41(4):243-265, 1919. MR 1506391

[10] Arthur B. Coble. Algebraic geometry and theta functions, volume 10 of American Mathematical Society Colloquium Publications. American Mathematical Society, Providence, R.I., 1982. Reprint of the 1929 edition. MR733252 (84m:14001)

[11] François R. Cossec and Igor V. Dolgachev. Smooth rational curves on Enriques surfaces. Math. Ann., 272(3):369-384, 1985. MR799668 (86m:14028)

[12] François R. Cossec and Igor V. Dolgachev. Enriques surfaces. I, volume 76 of Progress in Mathematics. Birkhäuser Boston Inc., Boston, MA, 1989. MR986969 (90h:14052) 
[13] Michel Demazure, Henry Charles Pinkham, and Bernard Teissier, editors. Séminaire sur les Singularités des Surfaces, volume 777 of Lecture Notes in Mathematics. Springer, Berlin, 1980. Held at the Centre de Mathématiques de l'École Polytechnique, Palaiseau, 1976-1977. MR.579026 (82d:14021)

[14] Vinay V. Deodhar. A note on subgroups generated by reflections in Coxeter groups. Arch. Math. (Basel), 53(6):543-546, 1989. MR1023969 (91c:20063)

[15] Igor V. Dolgachev. On automorphisms of Enriques surfaces. Invent. Math., 76(1):163-177, 1984. MR739632 (85j:14076)

[16] Igor V. Dolgachev. Infinite Coxeter groups and automorphisms of algebraic surfaces. In The Lefschetz centennial conference, Part I (Mexico City, 1984), volume 58 of Contemp. Math., pages 91-106. Amer. Math. Soc., Providence, RI, 1986. MR860406 (87j:14068)

[17] Igor V. Dolgachev. Reflection groups in algebraic geometry. Bull. Amer. Math. Soc. (N.S.), 45(1):1-60, 2008. MR2358376 (2009h:14001)

[18] Igor V. Dolgachev. Classical Algebraic Geometry: A Modern View, Cambridge Univ. Press, 2012, to appear.

[19] Igor V. Dolgachev. Cremona special sets of points in products of projective spaces. (English) In Ebeling, Wolfgang (ed.) et al., Complex and differential geometry. Conference held at Leibniz Universität Hannover, Germany, September 14-18, 2009. Proceedings. Berlin: Springer. Springer Proceedings in Mathematics 8, 115-134 (2011).

[20] Igor V. Dolgachev and David Ortland. Point sets in projective spaces and theta functions. Astérisque, 165, 1988. MR.1007155 (90i:14009)

[21] Igor V. Dolgachev and De-Qi Zhang. Coble rational surfaces. Amer. J. Math., 123:79-114, 2001. MR.1827278 (2002e:14061)

[22] Adrien Douady and John H. Hubbard. A proof of Thurston's topological characterization of rational functions. Acta Math., 171(2):263-297, 1993. MR1251582 (94j:58143)

[23] Marat H. Gizatullin. Rational G-surfaces. Izv. Akad. Nauk SSSR Ser. Mat., 44(1):110-144, 239, 1980. MR.563788 (81d:14020)

[24] Brian Harbourne. Automorphisms of K3-like rational surfaces. In Algebraic Geometry, Bowdoin, Part II, Brunswick, Maine, 1985, volume 46 of Proc. Symp. Pure Math., pages 17-28. Amer. Math. Soc., Providence, RI, 1987. MR927971 (89f:14044)

[25] Brian Harbourne. Rational surfaces with infinite automorphism group and no antipluricanonical curve. Proc. Amer. Math. Soc., 99(3):409-414, 1987. MR875372 (88a:14043)

[26] Robin Hartshorne. Algebraic geometry. Graduate Texts in Mathematics, No. 52. SpringerVerlag, New York, 1977. MR0463157 (57:3116)

[27] Seligmann Kantor. Theorie der endlichen Gruppen von eindeutigen Transformationen in der Ebene. Berlin, Mayer \& Müller, 1897.

[28] Martin Kneser. Quadratische Formen. Springer-Verlag, Berlin, 2002. MR2788987

[29] Max-Albert Knus. Quadratic and Hermitian forms over rings, volume 294 of Grundlehren der Math. Wiss. Springer-Verlag, Berlin, 1991. MR,1096299 (92i:11039)

[30] Max Lieblich and Davesh Maulik. On the cone conjecture for K3-surfaces in positive characteristic. arXiv:1102.3377, pages 1-8, 2011.

[31] Curtis T. McMullen. Dynamics on blowups of the projective plane. Publ. Math. Inst. Hautes Études Sci., (105):49-89, 2007. MR2354205 (2008m:37076)

[32] David R. Morrison. On K3 surfaces with large Picard number. Invent. Math., 75(1):105-121, 1984. MR728142 (85j:14071)

[33] David Mumford. Lectures on curves on an algebraic surface. Princeton Univ. Press, 1966. MR0209285 (35:187)

[34] Masayoshi Nagata. On rational surfaces. I. Irreducible curves of arithmetic genus 0 or 1. Mem. Coll. Sci. Univ. Kyoto Ser. A Math., 32:351-370, 1960. MR0126443(23:A3739)

[35] Masayoshi Nagata. On rational surfaces. II. Mem. Coll. Sci. Univ. Kyoto Ser. A Math., 33:271-293, 1960/1961. MR0126444(23:A3740)

[36] Viacheslav V. Nikulin. Integer symmetric bilinear forms and some of their geometric applications. Izv. Akad. Nauk SSSR Ser. Mat., 43(1):111-177, 238, 1979. MR525944 (80j:10031)

[37] Viacheslav V. Nikulin. Quotient-groups of groups of automorphisms of hyperbolic forms by subgroups generated by 2-reflections. algebro-geometric applications. J. Soviet Mathematics, 22:1401-1475, 1983.

[38] Viacheslav V. Nikulin. Elliptic fibrations on K3 surfaces. arXiv:1010.3904, pages 1-19, 2012. 
[39] Jean-Pierre Serre. Cours d'arithmétique. Lecture Notes in Mathematics. Presses Univ. de France, Paris, 1977. MR0498338 (58:16473)

[40] Tomoyuki Takenawa. Algebraic entropy and the space of initial values for discrete dynamical systems. J. Phys. A, 34(48):10533-10545, 2001. Symmetries and integrability of difference equations (Tokyo, 2000). MR:1877473(2002m:32026)

IRMAR, UMR 6625 Du CNRS et Université de Rennes 1, BÂt. 22-23 du Campus de Beaulieu, F-35042 Rennes Cedex; DMA, UMR 8553 du CNRS, École Normale Supérieure DE PARIS, 45 RUe D'Ulm, F-75230 PARIS CEDEX 05

E-mail address: serge.cantat@univ-rennes1.fr

Department of Mathematics, University of Michigan, 525 E. University Avenue, Ann Arbor, Michigan, 49109

E-mail address: idolga@umich.edu 